\title{
ILU PRECONDITIONERS FOR NONSYMMETRIC SADDLE-POINT MATRICES WITH APPLICATION TO THE INCOMPRESSIBLE NAVIER-STOKES EQUATIONS*
}

\author{
IGOR N. KONSHIN ${ }^{\dagger},{\text { MAXIM A. OLSHANSKII }{ }^{\ddagger}, \text { AND YURI V. VASSILEVSKI }}^{\S}$
}

\begin{abstract}
Motivated by the numerical solution of the linearized incompressible Navier-Stokes equations, we study threshold incomplete LU factorizations for nonsymmetric saddle-point matrices. The resulting preconditioners are used to accelerate the convergence of a Krylov subspace method applied to finite element discretizations of fluid dynamics problems in three space dimensions. The paper presents and examines an extension for nonsymmetric matrices of the Tismenetsky-Kaporin incomplete factorization. It is shown that in numerically challenging cases of higher Reynolds number flows one benefits from using this two-parameter modification of a standard threshold ILU preconditioner. The performance of the ILU preconditioners is studied numerically for a wide range of flow and discretization parameters, and the efficiency of the approach is shown if threshold parameters are chosen suitably. The practical utility of the method is further demonstrated for the haemodynamic problem of simulating blood flow in a right coronary artery of a real patient.
\end{abstract}

Key words. iterative methods, preconditioning, threshold ILU factorization, Navier-Stokes equations, finite element method, haemodynamics

AMS subject classifications. 65F10,65N22, 65F50

DOI. $10.1137 / 15 \mathrm{M} 1012311$

1. Introduction. This research is motivated by the numerical solution of the Navier-Stokes equations governing the flow of viscous incompressible Newtonian fluids. For a bounded domain $\Omega \subset \mathbb{R}^{d}(d=2,3)$ with boundary $\partial \Omega$, time interval $[0, T]$, and data $\mathbf{f}, \mathbf{g}$, and $\mathbf{u}_{0}$, the goal is to find a velocity field $\mathbf{u}=\mathbf{u}(\mathbf{x}, t)$ and pressure field $p=p(\mathbf{x}, t)$ such that

$$
\left\{\begin{aligned}
\frac{\partial \mathbf{u}}{\partial t}-\nu \Delta \mathbf{u}+(\mathbf{u} \cdot \nabla) \mathbf{u}+\nabla p=\mathbf{f} & \text { in } \Omega \times(0, T], \\
\operatorname{div} \mathbf{u}=0 & \text { in } \Omega \times[0, T], \\
\mathbf{u}=\mathbf{g} \text { on } \Gamma_{0} \times[0, T], \quad-\nu(\nabla \mathbf{u}) \cdot \mathbf{n}+p \mathbf{n}=\mathbf{0} & \text { on } \Gamma_{\mathrm{N}} \times[0, T], \\
\mathbf{u}(\mathbf{x}, 0)=\mathbf{u}_{0}(\mathbf{x}) & \text { in } \Omega,
\end{aligned}\right.
$$

where $\nu$ is the kinematic viscosity, $\Delta$ is the Laplacian, $\nabla$ is the gradient, and div is the divergence; $\partial \Omega=\bar{\Gamma}_{0} \cup \bar{\Gamma}_{\mathrm{N}}$ and $\Gamma_{0} \neq \varnothing$. Different flow regimes are characterized by the dimensionless Reynolds number $R e=\frac{U L}{\nu}$, where $U$ and $L$ are characteristic velocity and linear dimension. Solving (1.1) numerically is known to get harder for higher values of Re. Implicit time discretization and linearization of the Navier-Stokes

*Submitted to the journal's Methods and Algorithms for Scientific Computing section March 12, 2015; accepted for publication (in revised form) July 15, 2015; published electronically September 2, 2015. This work was supported by the Russian Science Foundation through the grant 14-31-00024 and numerical implementation and experiments in sections 5.1, 5.2 have been supported by RFBR grant 14-01-00830.

http://www.siam.org/journals/sisc/37-5/M101231.html

${ }^{\dagger}$ Institute of Numerical Mathematics, Institute of Nuclear Safety, Russian Academy of Sciences, Moscow, 119333, Russia (igor.konshin@gmail.com).

${ }^{\ddagger}$ Department of Mathematics, University of Houston, Houston, TX 77204 (molshan@math.uh. edu).

§Institute of Numerical Mathematics, Russian Academy of Sciences, Moscow Institute of Physics and Technology, Moscow, 117333 (yuri.vassilevski@gmail.com). 
system (1.1) by Picard fixed-point iteration result in a sequence of (generalized) Oseen problems of the form

$$
\left\{\begin{aligned}
\alpha \mathbf{u}-\nu \Delta \mathbf{u}+(\mathbf{w} \cdot \nabla) \mathbf{u}+\nabla p & =\hat{\mathbf{f}} & & \text { in } \Omega, \\
\operatorname{div} \mathbf{u} & =\hat{g} & & \text { in } \Omega, \\
\mathbf{u}=\mathbf{0} \text { on } \Gamma_{0}, & -\nu(\nabla \mathbf{u}) \cdot \mathbf{n}+p \mathbf{n}=\mathbf{0} & & \text { on } \Gamma_{\mathrm{N}},
\end{aligned}\right.
$$

where $\mathbf{w}$ is a known velocity field from a previous iteration or time step, $\alpha$ is proportional to the reciprocal of the time step ( $\alpha=0$ for a steady problem), and the right-hand side accounts for nonhomogenous boundary conditions in the nonlinear problem.

Finite element (FE) spatial discretization of (1.2) results in large, sparse systems of the form

$$
\left(\begin{array}{cc}
A & B^{T} \\
B & -C
\end{array}\right)\left(\begin{array}{l}
u \\
p
\end{array}\right)=\left(\begin{array}{c}
f \\
g
\end{array}\right)
$$

where $u$ and $p$ represent the discrete velocity and pressure, respectively, $A \in \mathbb{R}^{n \times n}$ is the discretization of the diffusion, convection, and time-dependent terms, $B^{T} \in$ $\mathbb{R}^{n \times m}$ is the discrete gradient, $B$ is the (negative) discrete divergence, $C \in \mathbb{R}^{m \times m}$ is a matrix resulting from possible pressure stabilization terms, and $f$ and $g$ contain forcing and boundary terms. If a discretization satisfies the LBB ("inf-sup") stability condition [15], no pressure stabilization is required and so $C=0$ holds. If the LBB condition is not satisfied, the stabilization matrix $C \neq 0$ is symmetric and positive semidefinite and the actual structure of $C$ depends on the particular FE pair being used. For a symmetric positive definite $A$, solving (1.3) is equivalent to finding the saddle point of a Lagrangian, and so the system (1.3) is often referred to as a saddlepoint system. In the literature, it is now common to refer to (1.3) as a nonsymmetric or generalized saddle-point system if $A \neq A^{T}$.

The efficient solution of systems of the form (1.3) necessitates rapidly convergent iterative methods. Thus, in the last decade, considerable work has been done in developing efficient preconditioners for Krylov subspace methods applied to incompressible flow problems; see the comprehensive treatments in $[3,12,29]$. It is typical for the preconditioning to exploit explicitly the block structure of the system (1.3). A popular approach builds upon preconditioners to the submatrix $A$ and pressure Schur complement matrix $S=B A^{-1} B^{T}+C$; see [13, 30, 43] for recent developments. Related to this class of methods are preconditioners based on the augmented Lagrangian reformulation of the saddle-point problem [5]. Block preconditioners based on an additive splitting include the Hermitian and skew-Hermitian splitting approach [2] and a dimensional split approach [4]. Constrained block preconditioners for nonsymmetric saddle-point matrices are treated in [7]. While the block preconditioners have proven to be effective in many cases, they are not yet completely robust with respect to variations of viscosity parameter, properties of advective velocity field $\mathbf{w}$, grid size, and anisotropy ratio. The discussion of geometric and algebraic multigrid preconditioners for the Oseen problem can be found in [41, 44]. For the assessment of block preconditioners in the haemodynamics context we refer to the recent paper [10].

An interesting alternative to block preconditioners for the Oseen problem is the preconditioning based on elementwise incomplete factorizations of the $2 \times 2$ block matrix from (1.3). Relatively little research is found in the literature on ILU preconditioners for the discrete Oseen system and, more general, for saddle-point linear 
algebraic systems. A review of incomplete Cholesky-type preconditioners applicable to symmetric saddle-point systems can be found in the recent reports [33, 34] (a symmetric system results from (1.2) if one sets $\mathbf{w}=0$ ). For nonsymmetric saddle-point systems that arise from the FE discretization of the incompressible Navier-Stokes equations the authors of $[8,42]$ developed ILU preconditioners, where the fill-in is allowed based on the connectivity of nodes rather than actual nonzeros in the matrix. The papers [35, 42] studied several reordering techniques for ILU factorization of (1.3) and found that some of the resulting preconditioners are competitive with the most advanced block preconditioners and are more straightforward to implement in standard FE codes.

The present paper focuses on incomplete LU factorizations with thresholds. As far as we are aware, threshold ILU factorizations for nonsymmetric saddle-point problems resulting from fluid dynamics applications have not been well studied in the literature. The present paper carries out a systematic study of $\operatorname{ILU}(\tau)$-type preconditioner performance and its dependence on the threshold parameter $\tau$, viscosity coefficient $\nu$, as well as on mesh discretization, and time step parameters. The properties of the advective velocity field $\mathbf{w}$ often also influence the performance of preconditioners, since the algebraic connectivity of nodes may be strongly influenced by local direction of flow. To assess the performance of ILU preconditioners, we experiment with unidirectional and complex three dimensional (3D) circulating flows including those arising in haemodynamics applications.

The paper also devises estimates for the LU factorization numerical stability for nonsymmetric saddle-point matrices. We show that if the $(1,1)$-block $A$ is a positive definite matrix, then the (exact) LU factorization of (1.3) exists and its numerical stability is determined by the ellipticity constant of $A$ and a quantity characterizing a ratio of symmetric and skew-symmetric parts of $A$. The analysis is applied to the discrete linearized Navier-Stokes equations and we discuss possible implications of this analysis for the stability of incomplete LU factorizations.

While in many situations $\operatorname{ILU}(\tau)$ with optimized $\tau$ provides inexpensive (in terms of fill-in) and efficient (in terms of iteration counts) preconditioners for (1.3), for higher Reynolds number flows (i.e., the case of small $\nu$ ) further developments are required. In such cases, we show that a two-parameter variant of the threshold ILU factorization $\operatorname{ILU}\left(\tau_{1}, \tau_{2}\right)$ may lead to a significant improvement. For symmetric positive definite matrices, this factorization is also known in the literature as the second order or Tismenetsky-Kaporin IC factorization. For both $\operatorname{ILU}(\tau)$ and $\operatorname{ILU}\left(\tau_{1}, \tau_{2}\right)$, the choice of optimal $\tau$ 's depends on problem parameters. Numerical experiments show that a choice of quasi-optimal parameters is feasible, leading to a preconditioner performance fairly insensitive to the variation of $\alpha$, grid anisotropy, complexity of $\mathbf{w}$, and depending mildly on $\nu$. Finally, we consider a test case of a flow in a digitally reconstructed right coronary artery of a real patient and for a set of parameters describing a physiologically relevant blood flow scenario. The paper reports on the performance of ILU preconditioners for this practical problem.

The remainder of the paper is organized as follows. In section 2 we give necessary details on the discretization method. Section 3 discusses LU factorizations for nonsymmetric saddle-point systems and its stability. Sufficient conditions on the existence of the $\mathrm{LU}$ factorization and an estimate on the entries of the LU factors are given here in terms of the properties of the $(1,1)$-block $A$. Further, this analysis is applied to the discretized system (1.2). Here sufficient conditions for positive definiteness of the $A$-block are derived. These conditions are sufficient for the existence 
of an LU factorization without pivoting. In section 4, we introduce a two-parameter Tismenetsky-Kaporin variant of the threshold ILU factorization for nonsymmetric nondefinite problems, which is used further for numerical experiments. In section 5 we consider two benchmark problems: a $3 \mathrm{D}$ flow in a cylindrical vessel and a $3 \mathrm{D}$ analog of the Beltrami flow proposed in [14]. For the discretization we use P2-P1 inf-sup stable FEs. For each of the problems we run experiments for a variety of physical and discretization parameters and on a sequence of refined tetrahedral discretizations. Conclusions are made about the performance of preconditioners and the suitable range of threshold parameters. Further we present results for the test case of a flow in a right coronary artery. Section 6 collects conclusions and a few closing remarks.

2. FE method. In this paper, we consider an inf-sup stable conforming FE method. To formulate it, we first need the weak formulation of the Oseen problem. Let $\mathbf{V}:=\left\{\mathbf{v} \in H^{1}(\Omega)^{3}:\left.\mathbf{v}\right|_{\Gamma_{0}}=\mathbf{0}\right\}$. Given $\mathbf{f} \in \mathbf{V}^{\prime}$, the problem is to find $\mathbf{u} \in \mathbf{V}$ and $p \in L^{2}(\Omega)$ such that

$$
\begin{aligned}
& \mathcal{L}(\mathbf{u}, p ; \mathbf{v}, q)=(\mathbf{f}, \mathbf{v})_{*}+(g, q) \quad \forall \mathbf{v} \in \mathbf{V}, q \in L^{2}(\Omega), \\
& \mathcal{L}(\mathbf{u}, p ; \mathbf{v}, q):=\alpha(\mathbf{u}, \mathbf{v})+\nu(\nabla \mathbf{u}, \nabla \mathbf{v})+((\mathbf{w} \cdot \nabla) \mathbf{u}, \mathbf{v})-(p, \operatorname{div} \mathbf{v})+(q, \operatorname{div} \mathbf{u}),
\end{aligned}
$$

where $(\cdot, \cdot)$ denotes the $L^{2}(\Omega)$ inner product and $(\cdot, \cdot)_{*}$ is the duality paring for $\mathbf{V}^{\prime} \times \mathbf{V}$.

We assume $T_{h}$ to be a collection of tetrahedra which is a consistent tetrahedrization of $\Omega$ satisfying the regularity condition

$$
\max _{\tau \in T_{h}} \operatorname{diam}(\tau) / \rho(\tau) \leq C_{T},
$$

where $\rho(\tau)$ is the diameter of a subscribed ball in $\tau$. A constant $C_{T}$ measures the maximum anisotropy ratio for $T_{h}$. Further we denote $h_{\min }=\min _{\tau \in T_{h}} \operatorname{diam}(\tau)$. Given conforming FE spaces $\mathbb{V}_{h} \subset \mathbf{V}$, and $\mathbb{Q}_{h} \subset L^{2}(\Omega)$, the Galerkin FE discretization of (1.2) is based on the weak formulation: Find $\left\{\mathbf{u}_{h}, p_{h}\right\} \in \mathbb{V}_{h} \times \mathbb{Q}_{h}$ such that

$$
\mathcal{L}\left(\mathbf{u}_{h}, p_{h} ; \mathbf{v}_{h}, q_{h}\right)=\left(\mathbf{f}, \mathbf{v}_{h}\right)_{*}+\left(g, q_{h}\right) \quad \forall \mathbf{v}_{h} \in \mathbb{V}_{h}, q_{h} \in \mathbb{Q}_{h} .
$$

In our experiments we shall use a P2-P1 Taylor-Hood FE pair, which satisfies the LBB compatibility condition for $\mathbb{V}_{h}$ and $\mathbb{Q}_{h}[15]$ and hence ensures well-posedness and full approximation order for the FE linear problem. If one enumerates velocity unknowns first and pressure unknowns next, then the resulting discrete system has the $2 \times 2$-block form (1.3) with $C=0$.

3. LU factorization and properties of $\boldsymbol{A}$ and $\boldsymbol{S}$. If the submatrices $A$ and $C$ of (1.3) are positive definite and positive semidefinite, respectively, the whole $2 \times 2$ block matrix is not sign definite. If $C=0$, its diagonal has zero entries. In general, $\mathrm{LU}$ factorization of such matrices requires pivoting (rows and columns permutations) for stability reasons. However, exploiting the block structure and the properties of blocks $A$ and $C$, one readily verifies that the $\mathrm{LU}$ factorization

$$
\mathcal{A}=\left(\begin{array}{cc}
A & B^{T} \\
B & -C
\end{array}\right)=\left(\begin{array}{cc}
L_{11} & 0 \\
L_{21} & L_{22}
\end{array}\right)\left(\begin{array}{cc}
U_{11} & U_{12} \\
0 & -U_{22}
\end{array}\right)
$$

with low (upper) triangle matrices $L_{11}, L_{22}\left(U_{11}, U_{22}\right)$ exists without pivoting, once $\operatorname{det}(A) \neq 0$ and there exist LU factorizations for the $(1,1)$-block

$$
A=L_{11} U_{11}
$$


and the Schur complement matrix $S:=B A^{-1} B^{T}+C$ is factorized as

$$
S=L_{22} U_{22} .
$$

To check (3.1), one lets $U_{12}=L_{11}^{-1} B^{T}$ and $L_{21}=B U_{11}^{-1}$.

An LU factorization of $A$ exists if the matrix is positive definite, however, its numerical stability (the relative size of entries in factors $L_{11}$ and $U_{11}$ ) may depend on how large is the skew-symmetric part of $A$ compared to the symmetric part. Indeed, denote $A_{\mathrm{S}}=\frac{1}{2}\left(A+A^{T}\right), A_{\mathrm{N}}=A-A_{\mathrm{S}}$ (we shall use similar notation for the symmetric and skew-symmetric parts of $S$ ). Denote by $\|\cdot\|_{F}$ the Frobenius matrix norm. Theorem 4.2.4 from [16] gives the bound on the size of elements of $L$ and $U$ :

$$
\left\|L_{11}\right\| U_{11} \mid \|_{F} \leq n\left(\left\|A_{\mathrm{S}}\right\|+\left\|A_{\mathrm{N}} A_{\mathrm{S}}^{-1} A_{\mathrm{N}}\right\|\right),
$$

where $|C|=\left\{\left|c_{i j}\right|\right\}$ for a matrix $C=\left\{c_{i j}\right\}$. Due to the symmetry and negative definiteness of $A_{\mathrm{N}} A_{\mathrm{S}}^{-1} A_{\mathrm{N}}$ it holds

$$
\left\|A_{\mathrm{N}} A_{\mathrm{S}}^{-1} A_{\mathrm{N}}\right\|=-\sup _{x \in \mathbb{R}^{n}} \frac{\left\langle A_{\mathrm{N}} A_{\mathrm{S}}^{-1} A_{\mathrm{N}} x, x\right\rangle}{\|x\|^{2}} .
$$

The positive definiteness of $A$ implies that $A_{S}$ is symmetric positive definite, and we make the substitution $x=A_{S}^{-\frac{1}{2}} x^{\prime}$ to get, after simple computations,

$$
\left\|A_{\mathrm{N}} A_{\mathrm{S}}^{-1} A_{\mathrm{N}}\right\|=\sup _{x \in \mathbb{R}^{n}} \frac{\left\|A_{\mathrm{S}}^{-\frac{1}{2}} A_{\mathrm{N}} A_{\mathrm{S}}^{-\frac{1}{2}} x\right\|^{2}}{\left\|A_{\mathrm{S}}^{-\frac{1}{2}} x\right\|^{2}} .
$$

Using $\left\|A_{\mathrm{S}}\right\| \leq\|A\|,\left\|A_{\mathrm{S}}^{\frac{1}{2}}\right\|\left\|A_{\mathrm{S}}^{-\frac{1}{2}} x\right\| \geq\|x\|$, and, for symmetric $A_{S}$, the identity $\left\|A_{\mathrm{S}}^{\frac{1}{2}}\right\|^{2}=\left\|A_{\mathrm{S}}\right\|$, we estimate

$$
\begin{aligned}
\left\|A_{\mathrm{N}} A_{\mathrm{S}}^{-1} A_{\mathrm{N}}\right\| & \leq \sup _{x \in \mathbb{R}^{n}} \frac{\left\|A_{\mathrm{S}}^{-\frac{1}{2}} A_{\mathrm{N}} A_{\mathrm{S}}^{-\frac{1}{2}} x\right\|^{2}\left\|A_{\mathrm{S}}^{\frac{1}{2}}\right\|^{2}}{\|x\|^{2}}=\left\|A_{\mathrm{S}}^{-\frac{1}{2}} A_{\mathrm{N}} A_{\mathrm{S}}^{-\frac{1}{2}}\right\|^{2}\left\|A_{\mathrm{S}}^{\frac{1}{2}}\right\|^{2} \\
& =\left\|A_{\mathrm{S}}^{-\frac{1}{2}} A_{\mathrm{N}} A_{\mathrm{S}}^{-\frac{1}{2}}\right\|^{2}\left\|A_{\mathrm{S}}\right\| \leq\left\|A_{\mathrm{S}}^{-\frac{1}{2}} A_{\mathrm{N}} A_{\mathrm{S}}^{-\frac{1}{2}}\right\|^{2}\|A\| .
\end{aligned}
$$

Hence, we deduce the following stability bound for the LU-factorization of the positive definite matrix $A$ :

$$
\frac{\left\|\left|L_{11}\left\|U_{11} \mid\right\|_{F}\right.\right.}{\|A\|} \leq n\left(1+\left\|A_{\mathrm{S}}^{-\frac{1}{2}} A_{\mathrm{N}} A_{\mathrm{S}}^{-\frac{1}{2}}\right\|^{2}\right) .
$$

The positive definiteness of $A$ implies that the Schur complement matrix is also positive definite, once $B^{T}$ has full column rank and $C \geq 0$. This is easy to see from the identities

$$
\langle S q, q\rangle=\langle B v, q\rangle+\langle C q, q\rangle=\left\langle v, B^{T} q\right\rangle+\langle C q, q\rangle=\langle A v, v\rangle+\langle C q, q\rangle,
$$

which are true for $q \in \mathbb{R}^{m}$ and $v:=A^{-1} B^{T} q \in \mathbb{R}^{n}$. Therefore, if $A$ is positive definite, then $S$ is also positive definite and the factorization $S=L_{22} U_{22}$ enjoys the stability bound similar to (3.2):

$$
\frac{\left\|L_{22}\right\| U_{22} \mid \|_{F}}{\|S\|} \leq m\left(1+\left\|S_{\mathrm{S}}^{-\frac{1}{2}} S_{\mathrm{N}} S_{\mathrm{S}}^{-\frac{1}{2}}\right\|^{2}\right) .
$$


Thus, in the case of a positive definite $(1,1)$-block, the quotients $\left\|A_{\mathrm{S}}^{-\frac{1}{2}} A_{\mathrm{N}} A_{\mathrm{S}}^{-\frac{1}{2}}\right\|$ and $\left\|S_{\mathrm{S}}^{-\frac{1}{2}} S_{N} S_{\mathrm{S}}^{-\frac{1}{2}}\right\|$ are largely responsible for the stability of the LU factorization for (1.3). The following lemma shows that it is sufficient to estimate $\left\|A_{\mathrm{S}}^{-\frac{1}{2}} A_{\mathrm{N}} A_{\mathrm{S}}^{-\frac{1}{2}}\right\|$.

Lemma 3.1. Let $A \in \mathbb{R}^{n \times n}$ be positive definite, then it holds

$$
\left\|S_{\mathrm{S}}^{-\frac{1}{2}} S_{N} S_{\mathrm{S}}^{-\frac{1}{2}}\right\| \leq\left\|A_{\mathrm{S}}^{-\frac{1}{2}} A_{\mathrm{N}} A_{\mathrm{S}}^{-\frac{1}{2}}\right\|=: C_{A} .
$$

Proof. Let $\widetilde{A}_{N}=A_{\mathrm{S}}^{-\frac{1}{2}} A_{\mathrm{N}} A_{\mathrm{S}}^{-\frac{1}{2}}$. We need the following identities [11]:

$$
\begin{aligned}
& \frac{1}{2}\left(A^{-1}+A^{-T}\right)=A_{\mathrm{S}}^{-\frac{1}{2}}\left(I-\widetilde{A}_{N}^{2}\right)^{-1} A_{\mathrm{S}}^{-\frac{1}{2}}, \\
& \frac{1}{2}\left(A^{-1}-A^{-T}\right)=A_{\mathrm{S}}^{-\frac{1}{2}}\left(I+\widetilde{A}_{N}\right)^{-1} \widetilde{A}_{N}\left(I-\widetilde{A}_{N}\right)^{-1} A_{\mathrm{S}}^{-\frac{1}{2}} .
\end{aligned}
$$

Note that due to the skew symmetry of $S_{\mathrm{S}}^{-\frac{1}{2}} S_{N} S_{\mathrm{S}}^{-\frac{1}{2}}$ it holds $|\lambda|=|\operatorname{Im}(\lambda)|$ for $\lambda \epsilon$ $\operatorname{sp}\left(S_{\mathrm{S}}^{-\frac{1}{2}} S_{N} S_{\mathrm{S}}^{-\frac{1}{2}}\right)$, where we use $\operatorname{sp}(\cdot)$ to denote the spectrum. We apply Bendixson's theorem [37] to estimate

$$
\begin{aligned}
\left\|S_{\mathrm{S}}^{-\frac{1}{2}} S_{N} S_{\mathrm{S}}^{-\frac{1}{2}}\right\| & =\max \left\{|\lambda|: \lambda \in \operatorname{sp}\left(S_{\mathrm{S}}^{-\frac{1}{2}} S_{N} S_{\mathrm{S}}^{-\frac{1}{2}}\right)\right\} \\
& =\max \left\{|\operatorname{Im}(\lambda)|: \lambda \in \operatorname{sp}\left(S_{\mathrm{S}}^{-\frac{1}{2}} S_{N} S_{\mathrm{S}}^{-\frac{1}{2}}\right)\right\} \\
& \leq \sup _{q \in \mathbb{C}^{m}} \frac{\left|\left\langle S_{N} q, q\right\rangle\right|}{\left\langle S_{\mathrm{S}} q, q\right\rangle} .
\end{aligned}
$$

Employing identities from (3.5), we can write

$$
\begin{aligned}
S_{\mathrm{S}} & =B A_{\mathrm{S}}^{-\frac{1}{2}}\left(I-\widetilde{A}_{N}^{*}\right)^{-1}\left(I-\widetilde{A}_{N}\right)^{-1} A_{\mathrm{S}}^{-\frac{1}{2}} B^{T}+C, \\
S_{N} & =B A_{\mathrm{S}}^{-\frac{1}{2}}\left(I-\widetilde{A}_{N}^{*}\right)^{-1} \widetilde{A}_{N}\left(I-\widetilde{A}_{N}\right)^{-1} A_{\mathrm{S}}^{-\frac{1}{2}} B^{T} .
\end{aligned}
$$

With the help of the substitution $v_{q}=\left(I-\widetilde{A}_{N}\right)^{-1} A_{\mathrm{S}}^{-\frac{1}{2}} B^{T} q$ in the right-hand side of (3.6) and recalling that $C$ is nonnegative definite, we obtain

$$
\left\|S_{\mathrm{S}}^{-\frac{1}{2}} S_{N} S_{\mathrm{S}}^{-\frac{1}{2}}\right\| \leq \sup _{q \in \mathbb{C}^{m}} \frac{\left|\left\langle\widetilde{A}_{N} v_{q}, v_{q}\right\rangle\right|}{\left\langle v_{q}, v_{q}\right\rangle+\langle C q, q\rangle} \leq \sup _{q \in \mathbb{C}^{m}} \frac{\left|\left\langle\widetilde{A}_{N} v_{q}, v_{q}\right\rangle\right|}{\left\|v_{q}\right\|^{2}} \leq\left\|\widetilde{A}_{N}\right\| .
$$

An estimate on the entries of $U_{12}$ and $L_{21}$ factors in (3.1) would form a complete picture of numerical stability of the factorization. The entries of these off-diagonal blocks can be estimated as follows. Using $\|A B\|_{F} \leq\|A\|\|B\|_{F}$ we get

$$
\left\|U_{12}\right\|_{F}=\left\|L_{11}^{-1} B^{T}\right\|_{F} \leq\left\|L_{11}^{-1}\right\|\left\|B^{T}\right\|_{F}=\left\|U_{11} A^{-1}\right\|\left\|B^{T}\right\|_{F} \leq\left\|U_{11}\right\|\left\|A^{-1}\right\|\left\|B^{T}\right\|_{F} .
$$

With the help of $(3.5)$ and noting $\left\|\left(I-\widetilde{A}_{N}\right)^{-1}\right\| \leq 1$ for a skew-symmetric $\widetilde{A}_{N}$, one also estimates

$$
\begin{aligned}
\left\|A^{-1}\right\| & \leq \frac{1}{2}\left(\left\|A^{-1}+A^{-T}\right\|+\left\|A^{-1}-A^{-T}\right\|\right) \\
& \leq\left\|A_{\mathrm{S}}^{-\frac{1}{2}}\right\|^{2}\left\|\left(I-\widetilde{A}_{N}^{2}\right)^{-1}\right\|+\left\|A_{\mathrm{S}}^{-\frac{1}{2}}\right\|^{2}\left\|\left(I-\widetilde{A}_{N}\right)^{-1}\right\|\left\|\left(I+\widetilde{A}_{N}\right)^{-1}\right\|\left\|\widetilde{A}_{N}\right\| \\
& \leq\left\|A_{\mathrm{S}}^{-\frac{1}{2}}\right\|^{2}\left(1+C_{A}\right)=\lambda_{\max }\left(A_{\mathrm{S}}^{-\frac{1}{2}}\right)^{2}\left(1+C_{A}\right)=\frac{1+C_{A}}{c_{A}}
\end{aligned}
$$


with the matrix $A$ ellipticity constant $c_{A}=\lambda_{\min }\left(A_{\mathrm{S}}\right)$. Repeating the same arguments for $\left\|L_{21}\right\|_{F}$, we arrive at the following bound:

$$
\frac{\left\|U_{12}\right\|_{F}+\left\|L_{21}\right\|_{F}}{\left(\left\|U_{11}\right\|+\left\|L_{11}\right\|\right)\|B\|_{F}} \leq \frac{m\left(1+C_{A}\right)}{c_{A}}
$$

The above analysis indicates that the LU factorization for (1.3) exists if the $(1,1)$ block $A$ is positive definite and the stability bounds depend on the constant $C_{A}$ which measures the ratio of skew symmetry for $A$ and the ellipticity constant $c_{A}$. In section 3.1 below, we estimate $C_{A}$ and $c_{A}$ for the discrete linearized Navier-Stokes system. In section 4, we argue why this analysis still of interest if one focuses on incomplete factorization.

3.1. Properties of $\boldsymbol{A}$ and $\boldsymbol{S}$. To study matrix properties, we invoke the FE formulation from section 2. Recall that we assume an inf-sup FE method, and so matrix $C$ is zero. The analysis below can be extended for stabilized elements $(C \neq 0)$, however, this makes arguments more technical and lengthy, since the properties of $C$ depend on the particular choice of stabilization parameters, which further depend on problem parameters. We avoid these extra details and treat only stable elements. Let $\left\{\varphi_{i}\right\}_{1 \leq i \leq n}$ and $\left\{\psi_{j}\right\}_{1 \leq j \leq m}$ be bases of $\mathbb{V}_{h}$ and $\mathbb{Q}_{h}$, respectively. For arbitrary $v \in \mathbb{R}^{n}$ and corresponding $\mathbf{v}_{h}=\sum_{i=1}^{n} v_{i} \varphi_{i}$, it holds

$$
\langle A v, v\rangle=\alpha\left\|\mathbf{v}_{h}\right\|^{2}+\nu\left\|\nabla \mathbf{v}_{h}\right\|^{2}+\frac{1}{2} \int_{\Gamma_{\mathrm{N}}}(\mathbf{w} \cdot \mathbf{n})\left|\mathbf{v}_{h}\right|^{2} d s+\frac{1}{2}\left((\operatorname{div} \mathbf{w}) \mathbf{v}_{h}, \mathbf{v}_{h}\right),
$$

where $\mathbf{n}$ is an outward normal on $\Gamma_{\mathrm{N}}$. We shall also need the velocity mass and stiffness matrices $M, K: M_{i j}=\left(\varphi_{i}, \varphi_{j}\right), K_{i j}=\left(\nabla \varphi_{i}, \nabla \varphi_{j}\right)$ and the pressure mass matrix $M_{p}:\left(M_{p}\right)_{i j}=\left(\psi_{i}, \psi_{j}\right)$.

While the first two terms on the right-hand side of (3.7) are positive, handling the rest of the terms requires some care. If $\Gamma_{\mathrm{N}}$ is an outflow part of the boundary, i.e., $(\mathbf{w} \cdot \mathbf{n})>0$, then the boundary integral is nonnegative. However, in practice, it is not uncommon when $(\mathbf{w} \cdot \mathbf{n})<0$ on a part of $\Gamma_{\mathrm{N}}$, and one likely can find such a $\mathbf{v}_{h}$ that the boundary integral in (3.7) is negative. Hence, we shall estimate this term using a $\mathrm{FE}$ trace inequality. We remark that modifications of boundary conditions from (1.1) on $\Gamma_{\mathrm{N}}$ are known, which insures that the resulting boundary integral is always nonnegative; see, e.g., [6]. Other artificial outflow boundary conditions, which lead to Dirichlet conditions in (1.2) on the entire boundary, are also common in fluid dynamics; see, e.g., $[28,32]$, in this case $\Gamma_{\mathrm{N}}=\varnothing$.

Next, if one assumes the incompressibility condition (second equation in (1.1)) to hold true for the advection velocity field $\mathbf{w}$, then the fourth term on the right-hand side vanishes. In practice, however, $\mathbf{w}$ is typically a $\mathrm{FE}$ velocity field, i.e., $\mathbf{w} \in \mathbb{V}_{h}$, which satisfies only weak divergence free constraint: $\left(\operatorname{div} \mathbf{w}, q_{h}\right)=0 \quad \forall q \in \mathbb{Q}_{h}$. For most stable FE for fluids and, in particular, for P2-P1 elements this weak divergence free equation does not imply div $\mathbf{w}=0$ pointwise (see $[18,27]$ and references therein for recent attempts to deal with this problem). Another possible way of getting rid of the (div w)-dependent term in (3.7) is to "skew symmetrize" the bilinear form by adding the consistent term $\frac{1}{2}\left((\operatorname{div} \mathbf{w}) \mathbf{u}_{h}, \mathbf{v}_{h}\right)$ to the FE formulation [39]. Otherwise the last term on the right-hand side of (3.7) should be controlled. We make the above conclusions more precise in Theorem 3.2 below. The theorem gives estimates on the ellipticity constant $c_{A}$ and the stability constant $C_{A}$ from (3.4). 
To formulate the result we need some preliminaries. First, recall the trace inequality

$$
\int_{\Gamma_{\mathrm{N}}}\left|\mathbf{v}_{h}\right|^{2} d s \leq C_{0}\left\|\nabla \mathbf{v}_{h}\right\|^{2} \quad \forall \mathbf{v}_{h} \in \mathbb{V}_{h}
$$

which allows control of the boundary term in (3.7) by the diffusion term, if $\nu$ is sufficiently large. To exploit the zero order term in (3.7), we need the FE trace and inverse inequalities

$$
\int_{\partial \tau} \mathbf{v}_{h}^{2} d s \leq C_{\mathrm{tr}} h_{\tau}^{-1}\left\|\mathbf{v}_{h}\right\|_{\tau}^{2}, \quad\left\|\nabla \mathbf{v}_{h}\right\|_{\tau} \leq C_{\mathrm{in}} h_{\tau}^{-1}\left\|\mathbf{v}_{h}\right\|_{\tau} \quad \forall \tau \in T_{h}, \mathbf{v}_{h} \in \mathbb{V}_{h}
$$

where the constants $C_{\mathrm{tr}}, C_{\text {in }}$ depend only on the polynomial degree $k$ and the shape regularity constant $C_{T}$ from (2.1). In addition, denote by $C_{\mathrm{f}}$ the constant from the Friedrichs inequality

$$
\left\|\mathbf{v}_{h}\right\| \leq C_{\mathrm{f}}\left\|\nabla \mathbf{v}_{h}\right\| \quad \forall \mathbf{v}_{h} \in \mathbb{V}_{h} .
$$

Let $C_{\mathbf{w}}:=\left\|(\mathbf{w} \cdot \mathbf{n})_{-}\right\|_{L^{\infty}\left(\Gamma_{\mathrm{N}}\right)}$.

To avoid the repeated use of generic but unspecified constants, in the remainder of the paper the binary relation $x \lesssim y$ means that there is a constant $c$ such that $x \leq c y$, and $c$ does not depend on the parameters which $x$ and $y$ may depend on, e.g., $\nu, \alpha$, mesh size, and properties of $\mathbf{w}$. Obviously, $x \gtrsim y$ is defined as $y \lesssim x$.

Theorem 3.2. Assume that $\mathbf{w} \in L^{\infty}(\Omega)$, and problem and discretization parameters satisfy

$$
\left\{\begin{array}{l}
C_{\mathbf{w}} C_{\mathrm{tr}} h_{\min }^{-1} \leq \frac{\alpha}{4} \quad \text { or } \quad C_{\mathbf{w}} C_{0} \leq \frac{\nu}{4} \\
\|\operatorname{div} \mathbf{w}\|_{L^{\infty}(\Omega)} \leq \frac{1}{4} \max \left\{\alpha, \nu C_{f}^{-1}\right\}
\end{array}\right.
$$

with constants defined in (3.8)-(3.10). Then the matrix $A$ is positive definite and it holds

$$
\langle A v, v\rangle \geq \frac{1}{4}\langle(\alpha M+\nu K) v, v\rangle \quad \forall v \in \mathbb{R}^{n} \quad \text { and } \quad C_{A} \lesssim 1+\frac{\|\mathbf{w}\|_{L^{\infty}(\Omega)}}{\sqrt{\nu \alpha}+\nu+h_{\min } \alpha},
$$

where $C_{A}$ is the constant defined in (3.4), and hence $c_{A} \geq \frac{1}{4} \lambda_{\min }(\alpha M+\nu K)$.

Furthermore, matrix $S:=B A^{-1} B^{T}$ is also positive definite and it holds

$$
\langle S q, q\rangle \gtrsim \frac{\left\langle M_{p} q, q\right\rangle}{\left(\nu+\alpha+\|\mathbf{w}\|_{L^{\infty}\left(\Gamma_{\mathrm{N}}\right)}+\|\operatorname{div} \mathbf{w}\|_{L^{\infty}(\Omega)}\right)\left(1+C_{A}^{2}\right)} \quad \forall q \in \mathbb{R}^{m} .
$$

Proof. Applying (3.8) and (3.9) in (3.7), we deduce

$$
\begin{gathered}
\langle A v, v\rangle \geq \alpha\left\|\mathbf{v}_{h}\right\|^{2}+\nu\left\|\nabla \mathbf{v}_{h}\right\|^{2}-\frac{C_{\mathbf{w}}}{2} \int_{\Gamma_{\mathrm{N}}}\left|\mathbf{v}_{h}\right|^{2} d s-\frac{1}{2}\|\operatorname{div} \mathbf{w}\|_{L^{\infty}(\Omega)}\left\|\mathbf{v}_{h}\right\|^{2} \\
\geq \alpha\left\|\mathbf{v}_{h}\right\|^{2}+\nu\left\|\nabla \mathbf{v}_{h}\right\|^{2}-\frac{C_{\mathbf{w}}}{2} \min \left\{C_{0}\left\|\nabla \mathbf{v}_{h}\right\|^{2}, C_{\mathrm{tr}} h_{\min }^{-1}\left\|\mathbf{v}_{h}\right\|^{2}\right\} \\
-\frac{1}{2}\|\operatorname{div} \mathbf{w}\|_{L^{\infty}(\Omega)}\left\|\mathbf{v}_{h}\right\|^{2} .
\end{gathered}
$$


To ensure the right-hand side is positive, we assume conditions (3.11) on problem parameters and coefficients. Employing conditions (3.11) in (3.13), we deduce

$$
\langle A v, v\rangle \geq \frac{1}{4}\left(\alpha\left\|\mathbf{v}_{h}\right\|^{2}+\nu\left\|\nabla \mathbf{v}_{h}\right\|^{2}\right)=\frac{1}{4}(\alpha\langle M v, v\rangle+\nu\langle K v, v\rangle) \quad \forall v \in \mathbb{R}^{n} .
$$

Further, we estimate

$$
\begin{aligned}
C_{A}:=\left\|A_{\mathrm{S}}^{-\frac{1}{2}} A_{\mathrm{N}} A_{\mathrm{S}}^{-\frac{1}{2}}\right\| & =\max \left\{|\lambda|: \lambda \in \operatorname{sp}\left(A_{\mathrm{S}}^{-\frac{1}{2}} A_{\mathrm{N}} A_{\mathrm{S}}^{-\frac{1}{2}}\right)\right\} \\
& =\max \left\{|\lambda|: \lambda \in \operatorname{sp}\left(A_{\mathrm{S}}^{-1} A_{\mathrm{N}}\right)\right\} \\
& \leq\left\|A_{\mathrm{S}}^{-1} A_{\mathrm{N}}\right\|_{*},
\end{aligned}
$$

and for $\|\cdot\|_{*}$ we choose a matrix norm induced by the vector norm $\langle(\alpha M+\nu K) \cdot, \cdot\rangle^{\frac{1}{2}}$. For a given $v \in \mathbb{R}^{n}$ and $u=A_{\mathrm{S}}^{-1} A_{\mathrm{N}} v$ consider their FE counterparts $\mathbf{v}_{h}, \mathbf{u}_{h} \in \mathbb{V}_{h}$. Then $A_{\mathrm{S}} u=A_{\mathrm{N}} v$ can be written in a FE form as

$$
\begin{aligned}
\nu\left(\nabla \mathbf{u}_{h}, \nabla \boldsymbol{\varphi}_{h}\right)+\alpha\left(\mathbf{u}_{h}, \boldsymbol{\varphi}_{h}\right)+ & \frac{1}{2} \int_{\Gamma_{\mathrm{N}}}(\mathbf{w} \cdot \mathbf{n}) \mathbf{u}_{h} \cdot \boldsymbol{\varphi}_{h} d s+\frac{1}{2}\left((\operatorname{div} \mathbf{w}) \mathbf{u}_{h}, \boldsymbol{\varphi}_{h}\right) \\
& =\frac{1}{2}\left[\left(\mathbf{w} \cdot \nabla \mathbf{v}_{h}, \boldsymbol{\varphi}_{h}\right)-\left(\mathbf{w} \cdot \nabla \boldsymbol{\varphi}_{h}, \mathbf{v}_{h}\right)\right] \quad \forall \boldsymbol{\varphi}_{h} \in \mathbb{V}_{h}
\end{aligned}
$$

We set $\varphi_{h}=\mathbf{u}_{h}$. For the left-hand side of (3.16) the lower bound (3.14) holds. To estimate the right-hand side, we apply the Cauchy-Schwarz inequality

$$
\left[\left(\mathbf{w} \cdot \nabla \mathbf{v}_{h}, \varphi_{h}\right)-\left(\mathbf{w} \cdot \nabla \boldsymbol{\varphi}_{h}, \mathbf{v}_{h}\right)\right] \leq\|\mathbf{w}\|_{L^{\infty}(\Omega)}\left(\left\|\mathbf{u}_{h}\right\|\left\|\nabla \mathbf{v}_{h}\right\|+\left\|\nabla \mathbf{u}_{h}\right\|\left\|\mathbf{v}_{h}\right\|\right) .
$$

Further we estimate terms on the right-hand side by employing Young's, Friedrichs, and FE inverse inequalities. Thus, one can estimate the product $\left\|\mathbf{u}_{h}\right\|\left\|\nabla \mathbf{v}_{h}\right\|$ in three different ways:

$$
\begin{aligned}
& \|\mathbf{w}\|_{L^{\infty}(\Omega)}\left\|\mathbf{u}_{h}\right\|\left\|\nabla \mathbf{v}_{h}\right\| \leq \frac{1}{16} \alpha\left\|\mathbf{u}_{h}\right\|^{2}+4\|\mathbf{w}\|_{L^{\infty}(\Omega)} \frac{1}{\alpha \nu}\left(\nu\left\|\nabla \mathbf{v}_{h}\right\|^{2}\right), \\
& \|\mathbf{w}\|_{L^{\infty}(\Omega)}\left\|\mathbf{u}_{h}\right\|\left\|\nabla \mathbf{v}_{h}\right\| \leq \frac{1}{16} \nu\left\|\nabla \mathbf{u}_{h}\right\|^{2}+4\|\mathbf{w}\|_{L^{\infty}(\Omega)} \frac{C_{\mathrm{f}}^{2}}{\nu^{2}}\left(\nu\left\|\nabla \mathbf{v}_{h}\right\|^{2}\right), \\
& \|\mathbf{w}\|_{L^{\infty}(\Omega)}\left\|\mathbf{u}_{h}\right\|\left\|\nabla \mathbf{v}_{h}\right\| \leq \frac{1}{16} \alpha\left\|\mathbf{u}_{h}\right\|^{2}+4\|\mathbf{w}\|_{L^{\infty}(\Omega)} \frac{C_{\mathrm{in}}^{2}}{\alpha^{2} h_{\min }^{2}}\left(\alpha\left\|\mathbf{v}_{h}\right\|^{2}\right) .
\end{aligned}
$$

Combining all three estimates gives

$$
\begin{aligned}
\|\mathbf{w}\|_{L^{\infty}(\Omega)} & \left\|\nabla \mathbf{v}_{h}\right\|\left\|\mathbf{u}_{h}\right\| \leq \frac{1}{16}\left(\nu\left\|\nabla \mathbf{u}_{h}\right\|^{2}+\alpha\left\|\mathbf{u}_{h}\right\|^{2}\right) \\
& +4\|\mathbf{w}\|_{L^{\infty}(\Omega)}^{2} \min \left\{\frac{1}{\alpha \nu}, \frac{C_{\mathrm{f}}^{2}}{\nu^{2}}, \frac{C_{\mathrm{in}}^{2}}{\alpha^{2} h_{\min }^{2}}\right\}\left(\nu\left\|\nabla \mathbf{v}_{h}\right\|^{2}+\alpha\left\|\mathbf{v}_{h}\right\|^{2}\right) .
\end{aligned}
$$

Using the same argument to treat the second term on the right-hand side of (3.17), we arrive at

$$
\begin{aligned}
\|\mathbf{w}\|_{L^{\infty}(\Omega)} \| & \left\|\mathbf{u}_{h}\right\|\left\|\mathbf{v}_{h}\right\| \leq \frac{1}{16}\left(\nu\left\|\nabla \mathbf{u}_{h}\right\|^{2}+\alpha\left\|\mathbf{u}_{h}\right\|^{2}\right) \\
& +4\|\mathbf{w}\|_{L^{\infty}(\Omega)}^{2} \min \left\{\frac{1}{\alpha \nu}, \frac{C_{\mathrm{in}}^{2}}{\alpha^{2} h_{\min }^{2}}, \frac{C_{\mathrm{f}}^{2}}{\nu^{2}}\right\}\left(\nu\left\|\nabla \mathbf{v}_{h}\right\|^{2}+\alpha\left\|\mathbf{v}_{h}\right\|^{2}\right) .
\end{aligned}
$$


For $\boldsymbol{\varphi}_{h}=\mathbf{u}_{h}$, the left-hand side of (3.16) equals $\langle A u, u\rangle$ and due to (3.14) it is estimated from below by $\nu\left\|\nabla \mathbf{u}_{h}\right\|^{2}+\alpha\left\|\mathbf{u}_{h}\right\|^{2}$, while (3.17)-(3.19) give the upper bound for the right-hand side of (3.16). Hence, we derive using $\min \left\{a_{1}, a_{2}, a_{3}\right\} \leq$ $3\left(a_{1}^{-1}+a_{2}^{-1}+a_{3}^{-1}\right)^{-1}$, the estimate

$$
\nu\left\|\nabla \mathbf{u}_{h}\right\|^{2}+\alpha\left\|\mathbf{u}_{h}\right\|^{2} \lesssim\left(1+\frac{\|\mathbf{w}\|_{L^{\infty}(\Omega)}^{2}}{\nu \alpha+\nu^{2}+h_{\min }^{2} \alpha^{2}}\right)\left(\nu\left\|\nabla \mathbf{v}_{h}\right\|^{2}+\alpha\left\|\mathbf{v}_{h}\right\|^{2}\right) .
$$

Therefore, we proved

$$
C_{A}:=\left\|A_{\mathrm{S}}^{-\frac{1}{2}} A_{\mathrm{N}} A_{\mathrm{S}}^{-\frac{1}{2}}\right\| \leq\left\|A_{\mathrm{S}}^{-1} A_{\mathrm{N}}\right\|_{*} \lesssim\left(1+\frac{\|\mathbf{w}\|_{L^{\infty}(\Omega)}}{\sqrt{\nu \alpha}+\nu+h_{\min } \alpha}\right) .
$$

Denote $\tilde{c}_{\mathbf{w}}:=\|\mathbf{w}\|_{L^{\infty}\left(\Gamma_{\mathrm{N}}\right)}, \hat{c}_{\mathbf{w}}=\|\operatorname{div} \mathbf{w}\|_{L^{\infty}(\Omega)}$. To show the ellipticity estimate for the Schur complement matrix, we note that (3.7), (3.8), (3.10), and the LBB stability of the FE spaces yield the following relations:

$$
\begin{aligned}
\left\langle B A_{\mathrm{S}}^{-1}\right. & \left.B^{T} q, q\right\rangle=\sup _{v \in \mathbb{R}^{n}} \frac{\langle B v, q\rangle^{2}}{\left\langle A_{\mathrm{S}} v, v\right\rangle} \\
& \geq \sup _{\mathbf{v}_{h} \in \mathbb{V}_{h}} \frac{\left(\operatorname{div} \mathbf{v}_{h}, q_{h}\right)^{2}}{\nu\left\|\nabla \mathbf{v}_{h}\right\|^{2}+\alpha\left\|\mathbf{v}_{h}\right\|^{2}+C_{0} \tilde{c}_{\mathbf{w}}\left\|\nabla \mathbf{v}_{h}\right\|^{2}+\hat{c}_{\mathbf{w}}\left\|\mathbf{v}_{h}\right\|^{2}} \\
& \gtrsim \sup _{\mathbf{v}_{h} \in \mathbb{V}_{h}} \frac{\left(\operatorname{div} \mathbf{v}_{h}, q_{h}\right)^{2}}{\left(\nu+\alpha+\tilde{c}_{\mathbf{w}}+\hat{c}_{\mathbf{w}}\right)\left\|\nabla \mathbf{v}_{h}\right\|^{2}} \gtrsim \frac{\left\|q_{h}\right\|^{2}}{\nu+\alpha+\tilde{c}_{\mathbf{w}}+\hat{c}_{\mathbf{w}}} \\
& =\frac{\left\langle M_{p} q, q\right\rangle}{\nu+\alpha+\tilde{c}_{\mathbf{w}}+\hat{c}_{\mathbf{w}}} .
\end{aligned}
$$

With the help of the first identity from (3.5) and (3.21) we obtain

$$
\begin{aligned}
\langle S q, q\rangle & =\left\langle A^{-1} B^{T} q, B^{T} q\right\rangle=\left\langle\left(I-\left(A_{\mathrm{S}}^{-\frac{1}{2}} A_{\mathrm{N}} A_{\mathrm{S}}^{-\frac{1}{2}}\right)^{2}\right)^{-1} A_{\mathrm{S}}^{-\frac{1}{2}} B^{T} q, A_{\mathrm{S}}^{-\frac{1}{2}} B^{T} q\right\rangle \\
& \geq \frac{\left\langle A_{\mathrm{S}}^{-\frac{1}{2}} B^{T} q, A_{\mathrm{S}}^{-\frac{1}{2}} B^{T} q\right\rangle}{1+\left\|\left(A_{\mathrm{S}}^{-\frac{1}{2}} A_{\mathrm{N}} A_{\mathrm{S}}^{-\frac{1}{2}}\right)^{2}\right\|}=\frac{\left\langle B A_{\mathrm{S}}^{-1} B^{T} q, q\right\rangle}{1+\left\|\left(A_{\mathrm{S}}^{-\frac{1}{2}} A_{\mathrm{N}} A_{\mathrm{S}}^{-\frac{1}{2}}\right)^{2}\right\|} \\
& \gtrsim \frac{1}{\left(\nu+\alpha+\tilde{c}_{\mathrm{w}}+\hat{c}_{\mathrm{w}}\right)\left(1+\left\|\left(A_{\mathrm{S}}^{-\frac{1}{2}} A_{\mathrm{N}} A_{\mathrm{S}}^{-\frac{1}{2}}\right)\right\|^{2}\right)}\left\langle M_{p} q, q\right\rangle .
\end{aligned}
$$

Now we combine (3.22) and (3.20) to show the desired ellipticity estimate for $S$.

We are in position to discuss conditions (3.11), which guarantee the matrices $A$ and $S$ to be positive definite and so the saddle-point matrix admits LU factorization without pivoting. The first condition in (3.11) is effective only if $\Gamma_{\mathrm{N}} \neq \varnothing$. Also if the entire $\Gamma_{\mathrm{N}}$ is outflow boundary then $C_{\mathbf{w}}=0$ and the condition is trivially satisfied. Otherwise, either the Reynolds number should be sufficiently small (creeping flows) or a Courant-type condition $(\Delta t) \leq c h_{\min }$ should hold with a problem-dependent constant $c$ (we recall that $\alpha \approx(\Delta t)^{-1}$ ). From the first look, the second condition in (3.11) is not restrictive. For example, for P2-P1 Taylor-Hood elements and a second order time discretization, the FE velocity gradient converges quadratically to the one of true solution, and hence one may expect that $\|\operatorname{div} \mathbf{w}\|_{L^{\infty}(\Omega)} \leq \tilde{C}\left(h^{2}+(\Delta t)^{2}\right)$. This would make the left-hand side of the second condition small. On the other hand, the constant $\tilde{C}$ is data dependent, and for $\nu$ small enough the constant can be large. Fortunately, for any fixed unsteady problem one can choose such small $\Delta t$ that the second condition holds due to $\alpha \sim(\Delta t)^{-1}$. 
4. A two-parameter threshold ILU factorization. In this section we proceed with incomplete LU factorizations of (1.3). A few remarks are in order.

Any threshold incomplete factorization can be written in the form $A=L U-E$, with an error matrix $E$. How small the matrix $E$ is ruled by a threshold parameter $\tau>$ 0 . The error matrix $E$ largely defines the quality of preconditioning; see, for example, [21] for estimates on GMRES method convergence written in terms of $\|E\|$ and subject to a proper prescaling of $A$ and the diagonalizability assumption. Furthermore, if $A$ is positive definite, then there exists such a small $\tau$ that $L U$ is also positive definite and so estimates from [16] can be applied to assess the numerical stability of the incomplete factorization. For $c_{A}=\lambda_{\min }\left(A_{\mathrm{S}}\right)$, a sufficient condition is $\tau<c_{A} n^{-1}$. Although in practice this estimate is often too pessimistic, for realistic $\tau$ and nonsymmetric matrices, nonpositive or close to zero pivots may be encountered, and breakdown of an algorithm may happen. A number of remedies have been proposed in the literature to deal with the problem of breakdown. A concise review of these techniques and further references can be found in [1]. Although most of the techniques were developed for the symmetric positive definite case, some of them can be applied to nonsymmetric matrices. These techniques are pivot modification, diagonal shifting, matrix scaling, unknowns reordering, and the Ajiz-Jennings modification. Among them, we found the matrix two-sided scaling to be the most important in our applications. We shall review this technique later in this section. Now let us look at the situation with ILU factorization for saddle-point matrices with positive definite $(1,1)$-block.

It was observed in $[33,45]$ for symmetric saddle-point systems that the block factorization as in (3.1) can be used to construct an incomplete factorization. One way to do this is first to compute an ILU factorization for the (1,1)-block, $A \approx \widetilde{L}_{11} \widetilde{U}_{11}$, set $\widetilde{U}_{12}=\widetilde{L}_{11}^{-1} B^{T}$ and $\widetilde{L}_{21}=B \widetilde{U}_{11}^{-1}$, and define $\widetilde{L}_{22}$ and $\widetilde{U}_{22}$ as incomplete factors for the inexact Schur complement:

$$
B\left(\widetilde{L}_{11} \widetilde{U}_{11}\right)^{-1} B^{T}+C \approx \widetilde{L}_{22} \widetilde{U}_{22} .
$$

As we noted before, $A>0$ implies $\widetilde{L}_{11} \widetilde{U}_{11}>0$, at least for sufficiently small $\tau$, and so the inexact Schur complement is also positive definite. In the present paper, we apply a global incomplete factorization of the matrix instead of the above blockwise factorization. We also avoid pivoting, i.e., we preserve the ordering when velocity unknowns are numbered before pressure unknowns, and we still observe stable factorizations.

Theorem 3.2 shows that for certain flow regimes the stability constant $C_{A}$ from (3.12) may become large and the ellipticity constant $c_{A}$ approaches zero, which means the nonsymmetric part of the matrix dominates over the symmetric one. Even for advanced threshold ILU factorizations this drives the threshold parameter $\tau$ to be smaller and hence increases the fill-in. Results of the next section demonstrate that exactly this behavior of the algorithm is observed in numerical experiments. To ameliorate the performance of the preconditioning in such extreme situations, we consider the two-parameter Tismenetsky-Kaporin variant of the threshold ILU factorization. The factorization was introduced and first studied in $[20,38,40]$ for the symmetric positive definite case. Below we consider an extension of the Tismenetsky-Kaporin factorization to the case of nonsymmetric and saddle-point matrices and give further motivation for it.

Given a matrix $A \in \mathbb{R}^{n \times n}$, consider the factorization of the form

$$
A=L U+L R_{u}+R_{\ell} U-E,
$$


where $R_{u}$ and $R_{\ell}$ are strictly upper and lower triangular matrices, while $U$ and $L$ are upper and lower triangular matrices, respectively. Given two small parameters $\tau_{1}$ and $\tau_{2}$, we shall assume that the off-diagonal elements of $U$ and $L$ are either zero or have absolute values greater than $\tau_{1}$, while the absolute values of $R_{\ell}$ and $R_{u}$ entries are either zero or belong to $\left(\tau_{2}, \tau_{1}\right]$; and $E$ is an error matrix with entries of order $O\left(\tau_{2}\right)$. We shall call (4.1) the $\operatorname{ILU}\left(\tau_{1}, \tau_{2}\right)$ factorization of A. Of course, a generic $\operatorname{ILU}(\tau)$ can be viewed as (4.1) with $R_{u}=R_{\ell}=0$ and $\tau_{1}=\tau_{2}=\tau$. The important improvement the two-parameter ILU factorization gives over a generic $\operatorname{ILU}(\tau)$ is that the fill-in of $L$ and $U$ is ruled by the first threshold parameter $\tau_{1}$, while the quality of the resulting preconditioner is mainly defined by $\tau_{2}$, once $\tau_{1}^{2} \lesssim \tau_{2}$ holds. Roughly speaking, taking $\tau_{2}=\tau_{1}^{2}:=\tau^{2}$ one expects the fill-in of $\operatorname{ILU}\left(\tau_{1}, \tau_{2}\right)$ to be similar to that of $\operatorname{ILU}(\tau)$, while the convergence of the preconditioned Krylov subspace method is better and asymptotically (for $\tau \rightarrow 0$ ) can be comparable to the one with the $\operatorname{ILU}\left(\tau^{2}\right)$ preconditioner. This statement is made more precise in [20] for symmetric positive definite matrices, where estimates on the eigenvalues and K-condition number of $L^{-1} A U^{-1}$ were derived with $L^{T}=U$ and $R_{\ell}^{T}=R_{u}$. The price one pays is that computing $L, U$ factors for $\operatorname{ILU}\left(\tau_{1}, \tau_{2}\right)$ is computationally more costly than for $\operatorname{ILU}\left(\tau_{1}\right)$, since intermediate calculations involve the entries of $R_{u}$. However, this factorization phase of $\operatorname{ILU}\left(\tau_{1}, \tau_{2}\right)$ is still less expensive than that of $\operatorname{ILU}\left(\tau_{2}\right)$. Note that (4.1) can be also viewed as the incomplete $\operatorname{ILU}\left(\tau_{2}\right)$ factorization of the matrix $A$ with the implicitly added $R_{\ell} R_{u}$ matrix:

$$
A+R_{\ell} R_{u}=\widehat{L} \widehat{U}-E,
$$

and further filtering of factors on the level of $\tau_{1}$,

$$
\widehat{L}=L+R_{\ell}, \quad \widehat{U}=U+R_{u},
$$

to get $L$ and $U$. In the symmetric case, the (implicitly) added matrix $R_{\ell} R_{u}$ is positive semidefinite making the $\operatorname{ILU}\left(\tau_{1}, \tau_{2}\right)$ factorization potentially more stable than $\operatorname{ILU}\left(\tau_{2}\right)$ applied to $A$.

Not much analysis of the decomposition (4.1) is known for a general nonsymmetric case. We note that the estimate (2.5) from [17] applied to the matrix

$$
\left(L+R_{\ell}\right)\left(U+R_{u}\right)=A+R_{\ell} R_{u}+E
$$

yields the low bound for the pivots of the (4.1) factorization

$$
\left|L_{i i} U_{i i}\right| \geq \min _{v \in \mathbb{R}^{n}} \frac{\left\langle\left(A+R_{\ell} R_{u}+E\right) v, v\right\rangle}{\|v\|^{2}} \geq c_{A}-\left\|R_{\ell} R_{u}\right\|-\|E\|
$$

with the ellipticity constant $c_{A}$ and the norms $\left\|R_{\ell} R_{u}\right\|,\|E\|$ proportional to $\tau_{1}^{2}$ and $\tau_{2}$, respectively. Thus the stability of the system solution with matrices $L$ and $U$ is ruled by the values of the second parameter and the square of the first parameter, while the fill-in is defined by $\tau_{1}$ rather than $\tau_{1}^{2}$. Using $\operatorname{ILU}\left(\tau_{1}, \tau_{2}\right)$ becomes important for the efficiency of the ILU preconditioning, when the problem setup is such that the estimates from Theorem 3.2 predict that the stability constant $C_{A}$ is large and $c_{A}$ is small.

Similarly to the situation with $\operatorname{ILU}(\tau)$ factorization, an $\operatorname{ILU}\left(\tau_{1}, \tau_{2}\right)$ factorization for the saddle-point matrix $\mathcal{A}$ can be built based on two-parameter factorizations (without pivoting) for the $(1,1)$ block

$$
A=L_{1} U_{1}+L_{1} R_{u 1}+R_{\ell 1} U_{1}-E_{1}
$$


and the inexact Schur complement matrix $\widetilde{S}=C+B\left[\left(L_{1}+R_{l 1}\right)\left(U_{1}+R_{u 1}\right)\right]^{-1} B^{T}$,

$$
\widetilde{S}=L_{2} U_{2}+L_{2} R_{u 2}+R_{\ell 2} U_{2}-E_{2} .
$$

For a matrix $G \in \mathbb{R}^{n \times m}$ and real $\tau \geq 0$ denote $\{G\}^{\tau} \in \mathbb{R}^{n \times m}$ with entries $\{G\}_{i j}^{\tau}=G_{i j}$ if $\left|G_{i j}\right| \geq \tau$, and $\{G\}_{i j}^{\tau}=0$ otherwise; let $[G]^{\tau}=G-\{G\}^{\tau}$. Given (4.2) and (4.3) one may check the following factorization for the saddle-point matrix $\mathcal{A}$ :

$$
\mathcal{A}=\mathcal{L U}+\mathcal{L R}_{u}+\mathcal{R}_{\ell} \mathcal{U}-\mathcal{E}
$$

with sparse block factors $\mathcal{L}$ and $\mathcal{U}$,

$$
\mathcal{L}=\left(\begin{array}{cc}
L_{1} & 0 \\
\left\{B\left(U_{1}+R_{u 1}\right)^{-1}\right\}^{\tau_{1}} & L_{2}
\end{array}\right), \quad \mathcal{U}=\left(\begin{array}{cc}
U_{1} & \left\{\left(L_{1}+R_{l 1}\right)^{-1} B^{T}\right\}^{\tau_{1}} \\
0 & -U_{2}
\end{array}\right),
$$

strictly upper triangle matrices $\mathcal{R}_{\ell}^{T}$ and $\mathcal{R}_{u}$,

$$
\mathcal{R}_{\ell}=\left(\begin{array}{cc}
R_{\ell 1} & 0 \\
{\left[B\left(U_{1}+R_{u 1}\right)^{-1}\right]^{\tau_{1}}} & R_{\ell 2}
\end{array}\right), \quad \mathcal{R}_{u}=\left(\begin{array}{cc}
R_{u 1} & {\left[\left(L_{1}+R_{l 1}\right)^{-1} B^{T}\right]^{\tau_{1}}} \\
0 & -R_{u 2}
\end{array}\right),
$$

and the error matrix

$$
\mathcal{E}=\left(\begin{array}{cc}
E_{1} & -R_{\ell 1}\left[\left(L_{1}+R_{l 1}\right)^{-1} B^{T}\right]^{\tau_{1}} \\
-\left[B\left(U_{1}+R_{u 1}\right)^{-1}\right]^{\tau_{1}} R_{u 1} & -E_{2}-\left[B\left(U_{1}+R_{u 1}\right)^{-1}\right]^{\tau_{1}}\left[\left(L_{1}+R_{l 1}\right)^{-1} B^{T}\right]^{\tau_{1}}
\end{array}\right) .
$$

If (4.2) and (4.3) are $\operatorname{ILU}\left(\tau_{1}, \tau_{2}\right)$ factorizations, then the formulas above show the existence of a Tismenetsky-Kaporin-type incomplete factorization of (1.3), with the error matrix having elements of order $O\left(\tau_{2}+\tau_{1}^{2}\right)$. In practice, we do not exploit the block form and neither matrix $\widetilde{S}$ nor factorization (4.3) are generated explicitly.

4.1. The algorithm. In what follows, the algorithm makes no specific use of the block structure of the matrix $\mathcal{A}$, but can formally be applied to a generic nonsymmetric $A \in \mathbb{R}^{n \times n}$ (for a general matrix it can fail, of course). Thus, for the sake of notation, we denote by $A$ below some given nonsymmetric square matrix, rather than the $(1,1)$ block of $\mathcal{A}$, and $n=\operatorname{dim}(A)$.

4.1.1. Two-sided scaling of $\boldsymbol{A}$. The derivation of the $\operatorname{ILU}\left(\tau_{1}, \tau_{2}\right)$ preconditioner in the SPD case assumes such a scaling of the matrix and unknowns that all diagonal elements are equal to 1; see [20]. Clearly, in a nonsymmetric case such scaling is not always possible. However, for the performance of the method, we found it very important to rescale a given matrix. Thus, we look for scaling vectors $\ell, r \in \mathbb{R}^{n}$ such that the matrix $A^{\prime}=\operatorname{diag}(\ell) A \operatorname{diag}(r)$ has nearly balanced Euclidean norms of rows and columns. To accomplish this task, we apply the Sinkhorn algorithm [36] to the nonnegative matrix $F=\left[a_{k j}^{2}\right]_{k j=1}^{n}$. The Sinkhorn method is an iterative algorithm. One iteration of the algorithm reads

$$
\begin{aligned}
& \operatorname{diag}\left(r^{(k+1)}\right)=\operatorname{diag}\left(F^{T} \ell^{(k)}\right)^{-1}, \\
& \operatorname{diag}\left(\ell^{(k+1)}\right)=\operatorname{diag}\left(F r^{(k+1)}\right)^{-1} .
\end{aligned}
$$

We use the starting vector $\ell^{(0)}$ of all ones. In all experiments in the next sections we performed 5 iterations to find the scaling vectors, before any incomplete factorization was computed. The importance of a proper two-sided scaling for a quality of ILU factorizations for nonsymmetric matrices is discussed in [21]; see also [9, 22, 25, 26]. 
If an incomplete factorization is computed for the scaled matrix $A^{\prime}$ so that $L^{\prime} U^{\prime} \approx$ $A^{\prime}$, the triangular factors for the original matrix have to be rescaled:

$$
L U \approx A, \quad L=(\operatorname{diag}(\ell))^{-1} L^{\prime}, \quad U=U^{\prime}(\operatorname{diag}(r))^{-1} .
$$

In what follows, we will refer to matrices $\operatorname{diag}(\ell)$ and $\operatorname{diag}(r)$ as the left and right scaling matrices $D_{L}$ and $D_{R}$, respectively.

4.1.2. Rowwise $\operatorname{ILU}\left(\tau_{1}, \tau_{2}\right)$ factorization. In general, a two-parameter threshold ILU factorization algorithm we are using is similar to that of RIC2 from [20]. The main difference with RIC2 is the extension of the method to nonsymmetric matrices. The algorithm uses a rowwise data structure for both $L$ and $U$ factors; it was suggested and implemented by Sergei Goreinov in the open source software [23, 24].

Assume that the input matrix $A \in \mathbb{R}^{n \times n}$ to be factorized is given in the compressed sparse row (CSR) format. Dropping for a moment the error matrix, consider the $(i+1)$ th step of the rowwise $\operatorname{ILU}\left(\tau_{1}, \tau_{2}\right)$ algorithm in the block-matrix form

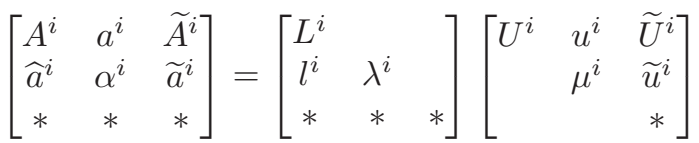

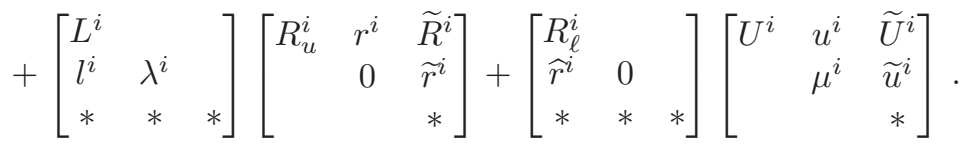

Here we use the convention to denote matrices and vectors (row or column) by Latin uppercase (capitals) and lowercase letters, respectively, and scalars by Greek symbols. All objects in the first row are known from the previous step, while $l^{i}, \lambda^{i}, \mu^{i}, \widetilde{u}^{i}$ have to be computed. The second row gives the set of equations

$$
\begin{aligned}
\widehat{a}^{i} & =\left(l^{i}+\widehat{r}^{i}\right) U^{i}+l^{i} R_{u}^{i}, \\
\alpha^{i} & =\left(l^{i}+\widehat{r}^{i}\right) u^{i}+l^{i} r^{i}+\lambda^{i} \mu^{i}, \\
\widetilde{a}^{i} & =\left(l^{i}+\widehat{r}^{i}\right) \widetilde{U}^{i}+l^{i} \widetilde{R}_{u}^{i}+\lambda^{i}\left(\widetilde{u}^{i}+\widetilde{r}^{i}\right) .
\end{aligned}
$$

Once one defines a rule for splitting a row vector $z=l^{i}+\widehat{r}^{i} \in \mathbb{R}^{i}$ into two structurally orthogonal parts $l^{i}$ and $\widehat{r}^{i}$ (i.e., $l_{k}^{i} \widehat{r}_{k}^{i}=0$ for $k=1, \ldots, i$ ), (4.6) is uniquely solvable for $l^{i}$ and $\widehat{r}^{i}$. The $\operatorname{ILU}\left(\tau_{1}, 0\right)$ method imposes the splitting $l_{k}^{i}=z_{k}$ if $\left|z_{k}\right|>\tau_{1}$, and $l_{k}^{i}=0$ otherwise. Recalling that $R_{u}^{i}$ is strictly upper triangle, the vectors $l^{i}$ and $\widehat{r}^{i}$ can be computed as is shown in steps (3)-(4) of the $\operatorname{ILU}\left(\tau_{1}, \tau_{2}\right)$ algorithm below, where vector $z$ is a part of a full size accumulator vector $v \in \mathbb{R}^{n}$.

After the vectors $l^{i}$ and $\widehat{r}^{i}$ are found, $\mu^{i}, \widetilde{u}^{i}$ can be computed from (4.6), (4.7) up to the scaling of $\lambda^{i}$ ( $\widetilde{u}^{i}$ is determined from the vector $\widetilde{z}=\widetilde{u}^{i}+\widetilde{r}^{i}$ using the same splitting rule). In our implementation, we set $\lambda^{i}=\left\|\widetilde{u}^{i}\right\|_{\ell^{\infty}}$. Finally, the entries of the factors not exceeding $\tau_{2}$ are dropped out and ignored in computations as in the standard threshold ILU strategy [31]. Pivots with absolute values smaller than $\tau_{2}$ are modified. The pseudocode of the resulting method is given below.

4.1.3. ILU $\left(\tau_{1}, \tau_{2}\right)$ algorithm pseudocode. Input: a sparse nonsymmetric matrix $A$, left and right scaling diagonal matrices $D_{L}$ and $D_{R}$ (see section 4.1.1), threshold parameters $0<\tau_{2} \leq \tau_{1}<1$. For a matrix $G, P(G)$ denotes the subset of indexes $(i, j)$ such that $G_{i j}=0$. Since $R_{\ell}$ is not computed in the course of the 
factorization, we use below the notation $R$ for the upper triangular error factor $R_{u}$; $v \in \mathbb{R}^{n}$ is an auxiliary vector initially set equal to 0 .

(1) Main loop by rows of $A$ to compute the rows of $L$ and $U$ :

for $i=1, \ldots, n$ :

(2) Initialize the row accumulator vector $v$ by the $i$ th row of the balanced matrix $A$ :

$$
\begin{aligned}
& \text { for } j=1, \ldots, n \text { and if }(i, j) \notin P(A): \\
& v_{j}:=\left(D_{L}\right)_{i i} a_{i j}\left(D_{R}\right)_{j j} \\
& \text { end for }
\end{aligned}
$$

(3) Loop over all already computed rows of $U$ :

for $k=1, \ldots, i-1$ and if $v_{k} \neq 0$ :

(4) Update the accumulator vector:

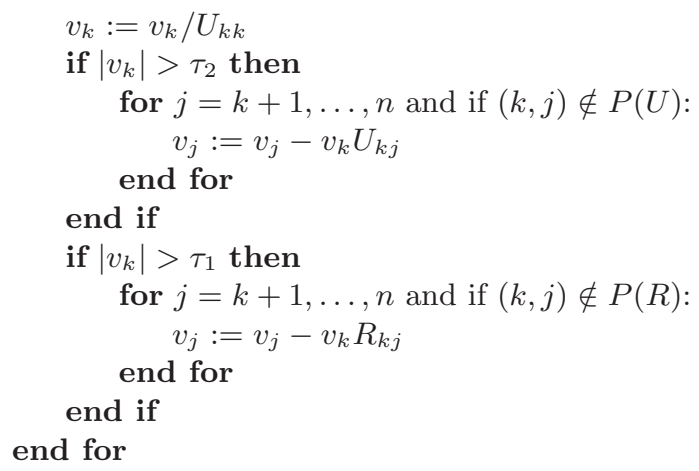

(5) Rescale the $i$ th row of $U$ :

$$
\begin{aligned}
& \lambda_{i}:=\max _{k=i, \ldots, n}\left|v_{k}\right| \\
& \text { if } \lambda_{i}<\tau_{2} \text { then } \\
& \quad \lambda_{i}:=\tau_{2} \\
& \text { end if } \\
& \text { for } j=i, \ldots, n \text { and if } v_{j} \neq 0: \\
& \quad v_{j}:=v_{j} / \lambda_{i} \\
& \text { end for }
\end{aligned}
$$

(6) Compute the $i$ th row of $L$ :

$$
\begin{aligned}
& L_{i i}=\lambda_{i} \\
& \text { for } j=1, \ldots, i-1 \text { and if }\left|v_{j}\right|>\tau_{1}: \\
& \quad L_{i j}:=v_{j}
\end{aligned}
$$

end for

(7) Compute the $i$ th row of $U$ and $R$ :

$$
\begin{aligned}
& \text { if }\left|v_{i}\right|<\tau_{2} \text { then } \\
& v_{i}:=\tau_{2} \cdot \operatorname{sign}\left(v_{i}\right) \\
& \text { end if } \\
& U_{i i}=v_{i} \\
& \text { for } j=i+1, \ldots, n \text { and if } v_{j} \neq 0 \text { : } \\
& \text { if }\left|v_{j}\right|>\tau_{1} \text { then } \\
& U_{i j}:=v_{j} \\
& \text { else if }\left|v_{j}\right|>\tau_{2} \text { then } \\
& \quad R_{i j}:=v_{j} \\
& \text { end if } \\
& \text { end for }
\end{aligned}
$$

(8) Clear nonzero elements of the row accumulator $v$ :

$$
\begin{aligned}
& \text { for } j=1, \ldots, n \text { and if } v_{j} \neq 0 \text { : } \\
& v_{j}:=0
\end{aligned}
$$

end for

end for 
(9) Perform the final rescaling of the incomplete factors $L$ and $U$ :

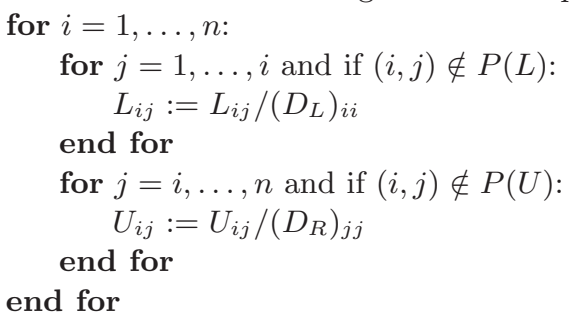

Note that the rowwise variant of the two-parameter ILU factorization drops off elements of matrix $R_{\ell}$ after processing the $i$ th row of $A$. This essentially saves the required working memory.

If an available working memory limit is exhausted in the course of computations, one can discard those entries of $R_{u}$ which are never used later in computations. Therefore, the factorization can be continued with (partially) compressed factors. In the present implementation of $\operatorname{ILU}\left(\tau_{1}, \tau_{2}\right)$, the sparsity of matrices is exploited as follows: $L$ and $U$ are stored in the CSR format using separate integer pointers. All the inner loops are made along the sparsity structure indices. Other loops over row accumulator vector $v$ are based on a linked-list data structure.

We remark that $\operatorname{ILU}\left(\tau_{1}, \tau_{2}\right)$ with $\tau_{1}=\tau_{2}$ is similar to the $\operatorname{ILUT}(\mathrm{p}, \tau)$ dual parameter incomplete factorization [31] with $p=n$ (all elements passing the threshold criterion are kept in the factors). If $\tau_{1}=\tau_{2}$ and no small pivots modification is done, the only differences between the algorithms are the scaling of pivots and a row-dependent scaling of threshold values used in ILUT.

5. Numerical results. In this section, we show the results of several numerical experiments with different values of fluid, discretization, and threshold parameters. We look for optimal values of ILU thresholds and how the preconditioner performance is sensitive to deviations of $\tau$ 's from these optimal values. The stopping criterion in all experiments is the decrease of the residual by 10 orders of magnitude. Three flow problems of increasing computational complexity are considered in this section.

5.1. Pipe flow. First, we consider a flow in a cylinder of circular cross section. The length of the cylinder is 2 , the diameter is $1, \mathbf{w}$ is the Poiseuille flow with $\max _{\Gamma_{0}}|\mathbf{w}|=1$. We prescribe zero no-slip conditions on the lateral boundary of the cylinder. The parabolic inflow profile is prescribed on the inlet of the cylinder and $-\nu(\nabla \mathbf{u}) \cdot \mathbf{n}+p \mathbf{n}=\mathbf{0}$ on the outlet.

To discretize the problem, we build several tetrahedral subdivisions of $\Omega$ (the lateral boundary is approximated by a triangulated surface). First, three increasingly fine meshes with regular tetrahadral elements are constructed. The corresponding number of degrees of freedom and average number of nonzero entries per row in the saddle-point matrix from (1.3) are the following: d.o.f. $=7330, \mathrm{nz}(A) / n=19.5$ (mesh 1); d.o.f. $=42066, \mathrm{nz}(A) / n=27.3$ (mesh 2); d.o.f. $=296715, \mathrm{nz}(A) / n=34.1$ (mesh 3 ). Further, two more meshes are built, each of these two contains 3 layers of anisotropic elements aligned along the lateral boundary. These two meshes mimic the situation when one has to adapt a mesh to a boundary layer. The data for these two meshes are given by d.o.f. $=501639, \mathrm{nz}(A) / n=37.0$, anisotropy ratio is equal to 5 (mesh 4); d.o.f. $=528598, \mathrm{nz}(A) / n=37.1$, anisotropy ratio is equal to $10($ mesh 5$)$. The latter mesh is illustrated in Figure 1.

In all experiments in this section, the resulting linear algebraic systems are solved by the preconditioned BiCGstab method with either $\operatorname{ILU}(\tau)$ or $\operatorname{ILU}\left(\tau_{1}, \tau_{2}\right)$ 

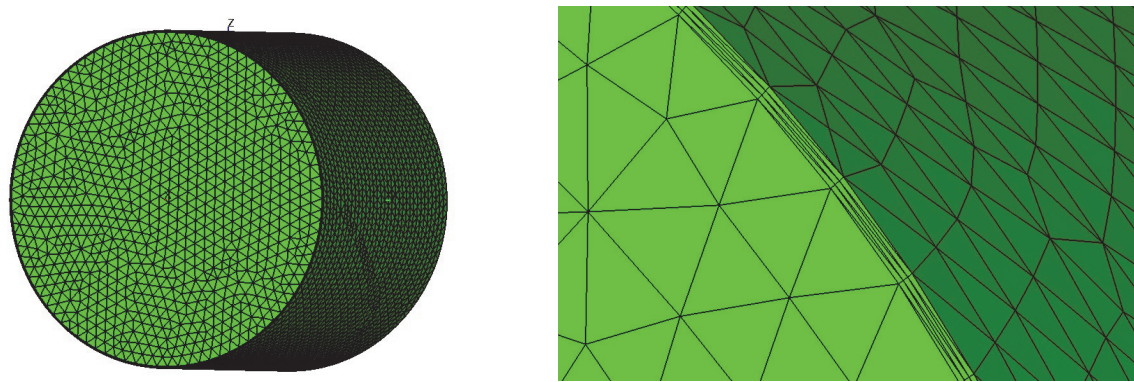

FIG. 1. Cylindrical domain with mesh 5 is shown on the left. The right picture zooms into the mesh near the lateral boundary to show anisotropic elemets, otherwise not seen on the left picture.

TABLE 1

The dependence of $I L U(\tau)$ performance on the choice of threshold parameter for the pipe flow; results are shown for $\nu=0.001, \alpha=1$, meshes 3 and 5 .

\begin{tabular}{l|rrrrr|rrrrr}
\hline$\tau$ & fill $_{L U}$ & \# it & \multicolumn{1}{c}{$T_{\text {build }}$} & \multicolumn{1}{c}{$T_{\text {it }}$} & \multicolumn{1}{c}{$T_{\mathrm{CPU}}$} & fill $_{L U}$ & \#it & $T_{\text {build }}$ & $T_{\text {it }}$ & $T_{\mathrm{CPU}}$ \\
\hline & & & Mesh 3 & & & & & Mesh 5 & & \\
0.100 & 0.387 & 135 & 1.2 & 13.5 & 14.7 & & & & & \\
0.080 & 0.497 & 94 & 1.5 & 10.2 & 11.7 & 0.385 & 129 & 2.3 & 23.3 & 25.6 \\
0.070 & 0.571 & 76 & 1.7 & 8.7 & 10.3 & 0.434 & 115 & 2.5 & 21.6 & 24.1 \\
0.060 & 0.667 & 60 & 1.9 & 7.3 & 9.2 & 0.519 & 69 & 2.9 & 13.7 & 16.6 \\
0.050 & 0.793 & 52 & 2.3 & 6.8 & 9.1 & 0.640 & 62 & 3.4 & 13.2 & 16.6 \\
0.040 & 0.969 & 49 & 2.9 & 7.0 & 9.9 & 0.798 & 52 & 4.2 & 12.1 & 16.4 \\
0.030 & 1.239 & 44 & 3.9 & 7.2 & 11.1 & 1.003 & 43 & 5.4 & 11.2 & 16.6 \\
0.020 & 1.722 & 30 & 5.9 & 5.9 & 11.8 & 1.360 & 31 & 7.7 & 9.5 & 17.3 \\
0.010 & 2.917 & 22 & 12.3 & 6.1 & 18.4 & 2.209 & 24 & 15.0 & 10.0 & 25.0 \\
0.007 & 3.754 & 18 & 17.8 & 5.9 & 23.8 & 2.766 & 18 & 21.0 & 8.7 & 29.7 \\
0.005 & 4.700 & 16 & 25.1 & 6.2 & 31.3 & 3.384 & 16 & 28.8 & 8.9 & 37.7 \\
0.003 & 6.472 & 13 & 41.6 & 6.3 & 47.9 & 4.520 & 12 & 46.5 & 8.2 & 54.7 \\
0.002 & 8.207 & 11 & 61.3 & 6.4 & 67.7 & 5.612 & 12 & 67.4 & 9.6 & 77.0 \\
0.001 & 11.954 & 9 & 115.5 & 7.0 & 122.5 & 8.007 & 10 & 125.4 & 10.6 & 135.9 \\
\hline
\end{tabular}

preconditioners, with zero initial guess. The $\operatorname{ILU}\left(\tau_{1}, \tau_{2}\right)$ preconditioner is computed by the algorithm from section 4.1.3, and $\operatorname{ILU}(\tau):=\operatorname{ILU}(\tau, \tau)$. All presented results are computed with 5 iterations to balance the matrix, as described in section 4.1.1. Using only 1 iteration we experienced slightly worse performance of the preconditioners. However, without the preprocessing both $\operatorname{ILU}(\tau)$ and $\operatorname{ILU}\left(\tau_{1}, \tau_{2}\right)$ fail for most of the examples treated in the numerical section.

In our first series of experiments, we study the $\tau$-dependence of the ILU $(\tau)$ preconditioner performance. The computations were run on the finest mesh 3 for $\nu=0.001$ and $\alpha=1$. The results are presented in Table $1 . T_{\text {build }}$ and $T_{\text {it }}$ show CPU time spent for building ILU factorization (including the two-sided scaling) and iterations, respectively; $T_{\mathrm{CPU}}=T_{\text {build }}+T_{\text {it }}$, and \#it is the number of BiCGstab iterations needed to satisfy the stoping criterion. The ratio of fill-in is computed from

$$
\text { fill }_{L U}=(\mathrm{nz}(L)+\mathrm{nz}(U)) / \mathrm{nz}(A), \quad \mathrm{nz}(A)=\sum_{i j} \operatorname{sign}\left|A_{i j}\right| .
$$

Note that fill $_{L U} \leq 1$ means that the number of nonzero elements in factors is less than in ILU(0), the commonly used ILU factorization by position. For smaller values of $\tau$ we observe the increase of fill-in and $T_{\text {build }}$, but the decrease of iteration numbers 

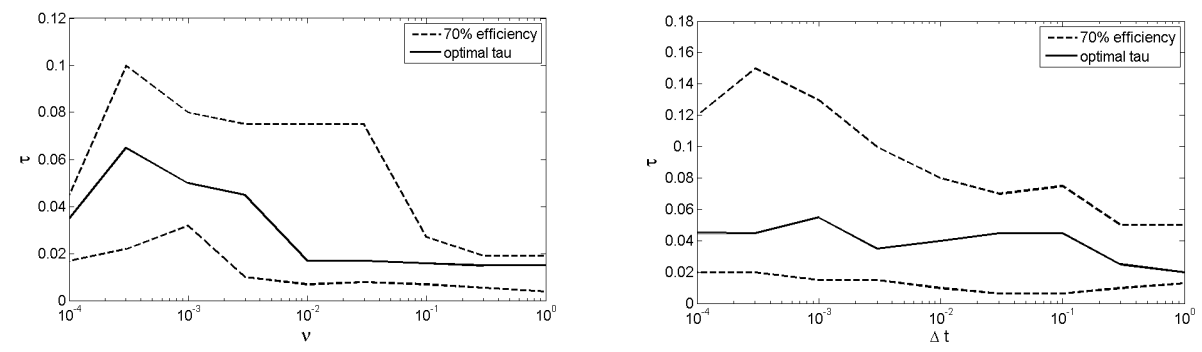

FIG. 2. The dependence of the optimal values of the threshold parameter $\tau$ in $I L U(\tau)$ on the viscosity $\nu$ (left) and $\alpha=\frac{1}{\Delta t}$ (right). Both plots also show the bounds on $\tau$ where the efficiency of the preconditioner is at least $70 \%$ of the optimal case.

and $T_{\mathrm{it}}$; both facts are expected. We repeated the same experiments with mesh 5 , which contains anisotropic elements. The results are shown in Table 1. We observe that the performance of the preconditioner does not change significantly, the optimal value of $\tau$ was found to be about the same. We run the same set of experiments with meshes 1,2, and 4, and observed that the optimal values of all $\tau$ 's are almost grid independent.

Further, we study the dependence of optimal threshold parameters with respect to the variation of $\nu$ and $\alpha$. The results are presented in Figure 2: on the left plot we vary $\nu$ for fixed $\alpha=10$ and given mesh 3, while on the right plot we vary $\alpha$ for fixed $\nu=0.01$ and the same mesh. Optimal $\tau$ 's were found with respect to total computational time, i.e., $T_{\mathrm{CPU}}=T_{\text {build }}+T_{\mathrm{it}}$. We also compute a range of "quasioptimal" $\tau$ 's, which is defined as the set of all parameters $\tau$ such that the efficiency of $\operatorname{ILU}(\tau)$ decreases at most by $30 \%$ compared to the case of the optimal value. From the plots we see that the optimal threshold values do depend on $\nu$ and $\alpha$. However, the range of acceptable values is rather wide, though it decreases for the diffusion dominated case. For further experiments, we choose a quasi-optimal value $\tau=0.03$, independent of parameters. Table 2 collects the results of experiments with this quasioptimal threshold value, showing the rate of fill-in, the number of iterations, and $T_{\mathrm{CPU}}$ for all five meshes, different $\nu$ 's, and $\alpha$ 's. One observes convergent iterations for all meshes and parameters, with a certain loss of performance for the strongly convection dominated Oseen problem discretized on strongly anisotropic mesh. It is interesting that a moderately convection dominated problem, i.e., $\nu \in\{0.1 ; 0.01 ; 0.001\}$, appears to be more amenable to efficient ILU preconditioning than a diffusion dominated case.

We repeat the same set of experiments, but now with the two-parameter ILU preconditioner. We set $\tau_{1}=0.03$ (equal to the quasi-optimal value in the $\operatorname{ILU}(\tau)$ preconditioner) and $\tau_{2}=c_{0} \tau_{1}^{2}$, with $c_{0}=7$. We note that in the symmetric positive definite case, the author of [20] recommends an ad hoc choice of $c_{0}=10$, while we found some decreasing of $c_{0}$ beneficial for the $\operatorname{ILU}\left(\tau_{1}, \tau_{2}\right)$ performance. The results are reported in Table 3 and they appear to be largely comparable to those obtained with $\operatorname{ILU}(\tau)$.

5.2. The Ethier-Steinman problem. Next we consider the well-known EthierSteinman solution for the Navier-Stokes equations from [14]. For chosen parameters 
TABLE 2

The performance of the one-parameter ILU( $\tau=0.03)$ preconditioner for the pipe flow test case. The results are shown for various values of viscosity $\nu, \alpha$, and different meshes.

\begin{tabular}{|c|c|c|c|c|c|c|c|c|c|c|c|}
\hline \multirow[b]{2}{*}{ Mesh } & $\nu:$ & \multicolumn{2}{|c|}{1} & \multicolumn{2}{|c|}{$10^{-1}$} & \multicolumn{2}{|c|}{$10^{-2}$} & \multicolumn{2}{|c|}{$10^{-3}$} & \multicolumn{2}{|c|}{$10^{-4}$} \\
\hline & $\alpha:$ & 10 & 100 & 10 & 100 & 10 & 100 & 10 & 100 & 10 & 100 \\
\hline & & \multicolumn{10}{|c|}{ fill $_{L U}$} \\
\hline 1 & & 0.88 & 0.73 & 0.74 & 0.80 & 0.80 & 1.06 & 1.06 & 1.18 & 1.17 & 1.20 \\
\hline 2 & & 0.89 & 0.78 & 0.78 & 0.62 & 0.72 & 0.94 & 1.26 & 1.19 & 1.71 & 1.24 \\
\hline 3 & & 0.89 & 0.85 & 0.85 & 0.66 & 0.72 & 0.72 & 1.24 & 1.08 & 2.86 & 1.25 \\
\hline 4 & & 0.89 & 0.86 & 0.86 & 0.74 & 0.77 & 0.71 & 1.00 & 0.92 & 1.83 & 1.14 \\
\hline 5 & & 0.83 & 0.81 & 0.80 & 0.70 & 0.73 & 0.73 & 1.00 & 0.99 & 1.91 & 1.02 \\
\hline & & \multicolumn{10}{|c|}{ \#it } \\
\hline 1 & & 12 & 10 & 9 & 12 & 11 & 14 & 13 & 15 & 15 & 15 \\
\hline 2 & & 48 & 21 & 19 & 19 & 19 & 25 & 23 & 26 & 27 & 26 \\
\hline 3 & & 170 & 61 & 59 & 34 & 32 & 38 & 44 & 42 & 79 & 52 \\
\hline 4 & & 169 & 62 & 56 & 34 & 31 & 43 & 41 & 67 & 87 & 73 \\
\hline 5 & & 177 & 67 & 59 & 36 & 32 & 50 & 43 & 59 & 99 & 136 \\
\hline & & \multicolumn{10}{|c|}{$T_{\mathrm{CPU}}$} \\
\hline 1 & & 0.04 & 0.04 & 0.04 & 0.05 & 0.05 & 0.05 & 0.05 & 0.05 & 0.06 & 0.06 \\
\hline 2 & & 0.82 & 0.44 & 0.42 & 0.38 & 0.42 & 0.58 & 0.71 & 0.68 & 1.00 & 0.70 \\
\hline 3 & & 25.1 & 10.2 & 10.0 & 5.86 & 5.91 & 7.15 & 11.0 & 9.33 & 33.2 & 11.8 \\
\hline 4 & & 43.8 & 18.2 & 16.8 & 10.7 & 10.3 & 13.0 & 15.6 & 21.0 & 44.2 & 25.1 \\
\hline 5 & & 51.4 & 19.6 & 17.7 & 11.4 & 10.7 & 15.3 & 16.9 & 20.5 & 51.8 & 41.9 \\
\hline
\end{tabular}

TABLE 3

The performance of the two-parameter $\operatorname{ILU}\left(\tau_{1}=0.03, \tau_{2}=7 \tau_{1}^{2}\right)$ preconditioner for the pipe flow test case. The results are shown for various values of viscosity $\nu, \alpha$, and different meshes.

\begin{tabular}{|c|c|c|c|c|c|c|c|c|c|c|c|}
\hline \multirow[b]{2}{*}{ Mesh } & \multirow{2}{*}{$\begin{array}{l}\nu: \\
\alpha:\end{array}$} & \multicolumn{2}{|c|}{1} & \multicolumn{2}{|c|}{$10^{-1}$} & \multicolumn{2}{|c|}{$10^{-2}$} & \multicolumn{2}{|c|}{$10^{-3}$} & \multicolumn{2}{|c|}{$10^{-4}$} \\
\hline & & 10 & 100 & 10 & 100 & 10 & 100 & 10 & 100 & 10 & 100 \\
\hline & & \multicolumn{10}{|c|}{ fill $_{L U}$} \\
\hline 1 & & 0.91 & 0.73 & 0.74 & 0.76 & 0.77 & 0.96 & 1.01 & 1.07 & 1.11 & 1.09 \\
\hline 2 & & 0.93 & 0.79 & 0.80 & 0.62 & 0.72 & 0.84 & 1.21 & 1.03 & 1.65 & 1.09 \\
\hline 3 & & 0.93 & 0.88 & 0.88 & 0.67 & 0.73 & 0.70 & 1.20 & 0.95 & 2.59 & 1.10 \\
\hline 4 & & 0.91 & 0.88 & 0.87 & 0.74 & 0.77 & 0.69 & 0.97 & 0.84 & 1.69 & 1.01 \\
\hline 5 & & 0.86 & 0.83 & 0.83 & 0.71 & 0.74 & 0.71 & 0.97 & 0.88 & 1.74 & 0.94 \\
\hline & & \multicolumn{10}{|c|}{ \#it } \\
\hline 1 & & 10 & 9 & 7 & 11 & 9 & 12 & 11 & 15 & 12 & 13 \\
\hline 2 & & 36 & 19 & 16 & 15 & 14 & 20 & 20 & 20 & 22 & 25 \\
\hline 3 & & 157 & 50 & 42 & 30 & 24 & 35 & 31 & 36 & 47 & 39 \\
\hline 4 & & 171 & 50 & 44 & 32 & 24 & 35 & 30 & 51 & 54 & 63 \\
\hline 5 & & 127 & 55 & 42 & 29 & 22 & 36 & 32 & 53 & 45 & 83 \\
\hline & & \multicolumn{10}{|c|}{$T_{\mathrm{CPU}}$} \\
\hline 1 & & 0.06 & 0.05 & 0.05 & 0.06 & 0.06 & 0.08 & 0.10 & 0.10 & 0.11 & 0.09 \\
\hline 2 & & 0.95 & 0.62 & 0.58 & 0.49 & 0.59 & 0.81 & 1.39 & 0.99 & 2.11 & 1.08 \\
\hline 3 & & 26.9 & 11.7 & 10.7 & 7.21 & 7.27 & 8.75 & 17.9 & 12.7 & 61.1 & 15.0 \\
\hline 4 & & 49.5 & 20.0 & 18.6 & 13.5 & 12.5 & 14.4 & 23.0 & 22.7 & 68.6 & 29.5 \\
\hline 5 & & 39.7 & 21.5 & 18.3 & 13.1 & 12.2 & 15.5 & 24.2 & 24.3 & 68.3 & 34.3 \\
\hline
\end{tabular}

$a, d$, and viscosity $\nu$, the exact solution is given in $[-1,1]^{3}$ by

$$
\begin{aligned}
& u_{1}=-a\left(e^{a x} \sin (a y+d z)+e^{a z} \cos (a x+d y)\right) e^{-\nu d^{2} t} \\
& u_{2}=-a\left(e^{a y} \sin (a z+d x)+e^{a x} \cos (a y+d z)\right) e^{-\nu d^{2} t} \\
& u_{3}=-a\left(e^{a z} \sin (a x+d y)+e^{a y} \cos (a z+d x)\right) e^{-\nu d^{2} t}
\end{aligned}
$$




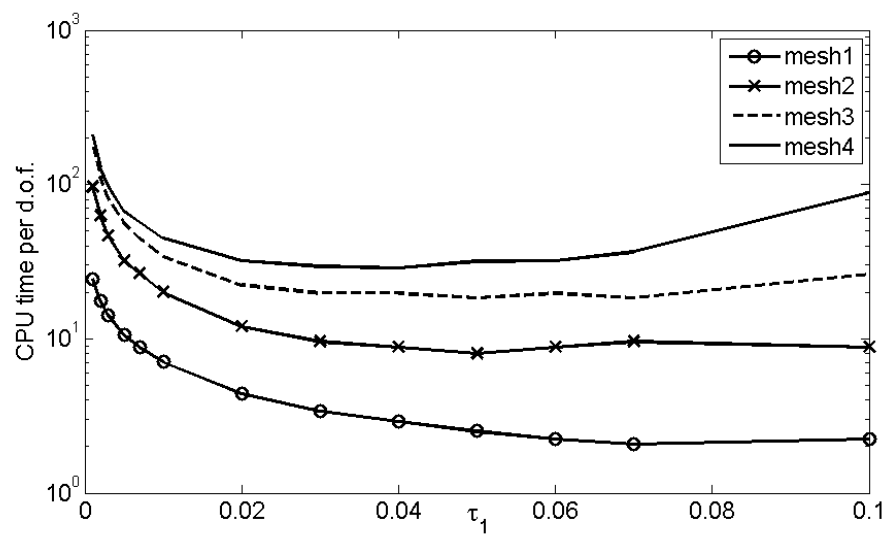

FIG. 3. Dependence of $I L U(\tau)$ on the threshold parameter $\tau$ for the Ethier-Steinman test case; $\nu=0.01, \alpha=10$.

and

$$
\begin{aligned}
p=-\frac{a^{2}}{2} & \left(e^{2 a x}+e^{2 a y}+e^{2 a z}+2 \sin (a x+d y) \cos (a z+d x) e^{a(y+z)}\right. \\
& +2 \sin (a y+d z) \cos (a x+d y) e^{a(z+x)} \\
& \left.+2 \sin (a z+d x) \cos (a y+d z) e^{a(x+y)}\right) e^{-2 \nu d^{2} t}
\end{aligned}
$$

In our experiments we set $a=\pi / 4, d=\pi / 2$, and vary $\nu$. This problem was developed as a 3D analog to the Taylor vortex problem, for the purpose of benchmarking. Although unlikely to be physically realized, it is a good test problem because it has an analytically known solution, but the flow has no principle direction and has a nontrivial vortical structure.

For the purpose of testing the algebraic solver, we do not perform time stepping, but linearize the Navier-Stokes equation over the analytical solution at $t=0.1$. For the discretization, a regular tetrahedrization of the cube $[-1,1]^{3}$ is built. The coarsest mesh is uniformly refined three times. This results in four gradually refined meshes. The corresponding number of degrees of freedom and average number of nonzero entries per row in the saddle-point matrix from (1.3) were the following: d.o.f. $=2251$, $\mathrm{nz}(A) / n=17.3$ (mesh 1); d.o.f. $=12420, \mathrm{nz}(A) / n=25.8$ (mesh 2); d.o.f. $=75660$, $\mathrm{nz}(A) / n=32.5$ (mesh 3); d.o.f. $=522220, \mathrm{nz}(A) / n=37.5$ (mesh 4). Similarly to the previous test, the resulting linear algebraic system was solved by the BiCGstab method with either $\operatorname{ILU}\left(\tau_{1}\right)$ or $\operatorname{ILU}\left(\tau_{1}, \tau_{2}\right)$ preconditioners and zero initial guess.

Figure 3 demonstrates the dependence of $\operatorname{ILU}(\tau)$ performance with respect to the choice of the threshold parameter $\tau$. The experiments were run with $\nu=0.01$, $\alpha=10$, and for all four meshes. The vertical axis shows the total CPU time per degree of freedom. We observe a definite dependence of optimal $\tau$ on the mesh size, but the range of quasi-optimal parameters is wide. We set $\tau=0.02$ and run the computation with $\operatorname{ILU}(\tau)$ and $\operatorname{ILU}\left(\tau, 7 \tau^{2}\right)$ for different values of viscosity coefficient $\nu \in\{1,0.1,0.01,0.001\}$ and parameter $\alpha \in\{1,10,100\}$. The results for two fine meshes are collected in Table 4 . From the results in this table, we see that in the range of moderate viscosity values, both preconditioners demonstrate very similar behavior with $\operatorname{ILU}(\tau)$ being somewhat cheaper during the setup phase. For the dif- 
TABLE 4

The performance of the $I L U(\tau=0.02)$ and $I L U\left(\tau_{1}=0.02, \tau_{2}=7 \tau_{1}^{2}\right)$ preconditioners for the Ethier-Steinman flow. The results are shown for various values of $\nu, \alpha$, and two different meshes.

\begin{tabular}{|c|c|c|c|c|c|c|c|c|c|c|c|c|c|}
\hline \multirow[b]{2}{*}{ Mesh } & \multicolumn{3}{|c|}{1} & \multicolumn{3}{|c|}{$10^{-1}$} & \multicolumn{3}{|c|}{$10^{-2}$} & \multicolumn{3}{|c|}{$10^{-3}$} & \multirow[b]{2}{*}{ |ILU } \\
\hline & $\alpha:$ & 10 & 100 & 1 & 10 & 100 & 1 & 10 & 100 & 1 & 10 & 100 & \\
\hline & \multicolumn{13}{|c|}{ fill $_{L U}$} \\
\hline 3 & 1.22 & 1.20 & 1.07 & 1.19 & 1.08 & 0.81 & 1.97 & 1.47 & 1.21 & 79.96 & 5.83 & 1.78 & $\operatorname{ILU}(\tau)$ \\
\hline 3 & 1.21 & 1.19 & 1.07 & 1.20 & 1.08 & 0.81 & 1.98 & 1.46 & 1.12 & 20.62 & 4.93 & 1.64 & $\operatorname{ILU}\left(\tau_{1}, \tau_{2}\right)$ \\
\hline 4 & 1.22 & 1.22 & 1.17 & 1.21 & 1.17 & 0.93 & 1.48 & 1.27 & 0.97 & $\mathrm{n} / \mathrm{c}$ & 6.53 & 1.89 & $\operatorname{ILU}(\tau)$ \\
\hline \multirow[t]{2}{*}{4} & 1.20 & 1.20 & 1.16 & 1.20 & 1.16 & 0.93 & 1.53 & 1.30 & 0.96 & 9.28 & 5.33 & 1.80 & $\operatorname{ILU}\left(\tau_{1}, \tau_{2}\right)$ \\
\hline & \multicolumn{13}{|c|}{ \#it(BCGstab) } \\
\hline 3 & 72 & 46 & 18 & 29 & 14 & 17 & 12 & 15 & 24 & $\mathrm{n} / \mathrm{c}$ & 38 & 28 & $\operatorname{ILU}(\tau)$ \\
\hline 3 & 58 & 37 & 16 & 24 & 14 & 13 & 12 & 14 & 15 & 58 & 19 & 17 & $\operatorname{ILU}\left(\tau_{1}, \tau_{2}\right)$ \\
\hline 4 & 337 & 296 & 50 & 119 & 38 & 26 & 27 & 22 & 31 & $\mathrm{n} / \mathrm{c}$ & 83 & 44 & $\operatorname{ILU}(\tau)$ \\
\hline 4 & 201 & 158 & 36 & 95 & 31 & 26 & 22 & 24 & 26 & 47 & 38 & 31 & $\operatorname{ILU}\left(\tau_{1}, \tau_{2}\right)$ \\
\hline \multicolumn{14}{|c|}{$T_{\mathrm{CPU}}($ BCGstab) } \\
\hline 3 & 4.4 & 3.4 & 1.5 & 2.2 & 1.4 & 1.3 & 2.1 & 1.7 & 1.8 & $\mathrm{n} / \mathrm{c}$ & 13.6 & 2.9 & $\operatorname{ILU}(\tau)$ \\
\hline 3 & 4.2 & 3.5 & 2.8 & 3.3 & 2.7 & 2.0 & 7.6 & 5.2 & 3.4 & 492 & 46.1 & 6.2 & $\operatorname{ILU}\left(\tau_{1}, \tau_{2}\right)$ \\
\hline 4 & 170.1 & 149.7 & 31.0 & 58.5 & 25.7 & 17.5 & 20.8 & 16.7 & 16.8 & $\mathrm{n} / \mathrm{c}$ & 234.1 & 38.4 & $\operatorname{ILU}(\tau)$ \\
\hline 4 & 93.8 & 96.9 & 35.7 & 69.9 & 35.1 & 26.8 & 51.2 & 42.8 & 35.1 & 2174 & 735 & 89.5 & $\operatorname{ILU}\left(\tau_{1}, \tau_{2}\right)$ \\
\hline \multicolumn{14}{|c|}{ \#it(GMRES(30)) } \\
\hline 3 & 67 & 54 & 29 & 41 & 25 & 26 & 22 & 26 & 34 & $\mathrm{n} / \mathrm{c}$ & 55 & 44 & $\operatorname{ILU}(\tau)$ \\
\hline 3 & 56 & 47 & 27 & 36 & 24 & 21 & 20 & 23 & 26 & 95 & 34 & 28 & $\operatorname{ILU}\left(\tau_{1}, \tau_{2}\right)$ \\
\hline 4 & 288 & 218 & 85 & 131 & 71 & 44 & 47 & 40 & 54 & $\mathrm{n} / \mathrm{c}$ & 128 & 64 & $\operatorname{ILU}(\tau)$ \\
\hline 4 & 157 & 126 & 63 & 98 & 57 & 41 & 41 & 38 & 41 & 62 & 58 & 46 & $\operatorname{ILU}\left(\tau_{1}, \tau_{2}\right)$ \\
\hline \multicolumn{14}{|c|}{$T_{\mathrm{CPU}}(\mathrm{GMRES}(30))$} \\
\hline 3 & 2.4 & 2.1 & 1.3 & 1.7 & 1.2 & 1.0 & 2.2 & 1.8 & 1.7 & $\mathrm{n} / \mathrm{c}$ & 12.6 & 2.7 & $\operatorname{ILU}(\tau)$ \\
\hline 3 & 3.4 & 3.1 & 2.3 & 2.9 & 2.3 & 1.7 & 6.6 & 4.5 & 3.2 & 472.9 & 41.7 & 5.6 & $\operatorname{ILU}\left(\tau_{1}, \tau_{2}\right)$ \\
\hline 4 & 69.9 & 54.9 & 25.5 & 36.1 & 22.6 & 14.4 & 22.0 & 18.1 & 17.7 & $\mathrm{n} / \mathrm{c}$ & 216.8 & 34.1 & $\operatorname{ILU}(\tau)$ \\
\hline 4 & 56.5 & 49.7 & 35.0 & 44.0 & 33.9 & 24.5 & 49.9 & 40.0 & 29.3 & 2207 & 668.5 & 81.4 & $\operatorname{ILU}\left(\tau_{1}, \tau_{2}\right)$ \\
\hline
\end{tabular}

fusion dominated case $(\nu=1, \alpha=1)$, when the matrix becomes more symmetric, the two-parameter preconditioning wins in terms of iteration number and total CPU time. The convection dominated case appears to be the hardest. Here $\operatorname{ILU}(\tau)$ fails for $\alpha=1$, while the usage of the two-parameter preconditioner leads to a convergent method. We repeated the same set of experiments with the restarted minimal residual method, GMRES(30). The performance of the solver turned out to be very similar to the preconditioned BiCGstab method, see Table 4.

Finally we have a closer look at the hardest case from Table 4, i.e., $\nu=0.001$ and $\alpha=1$, and experiment with different values of the threshold parameters. Table 5 shows the results of this experiment for $\nu=0.001$ and $\alpha=1$ on a fixed given mesh 3. We see that similar to the pipe flow case, the optimal parameter for $\operatorname{ILU}(\tau)$ decreases. Interestingly enough, the decrease of $\nu$ and $\alpha$ by 10 times resulted in the 10 times decrease of $\tau_{\mathrm{opt}}$, which is consistent with the ellipticity bound on matrix $A$ in Theorem 3.2. Also a "comfortable" zone around $\tau_{\text {opt }}$ shrinks making an overshoot in choosing the quasi-optimal $\tau$ easily possible. For this convection dominated problem, one clearly benefits from using the two-parameter ILU preconditioner. For two-parameter ILU, we fixed $\tau_{1}=0.02$ and vary the scaling factor $c_{0}$ in $\tau_{2}=c_{0} \tau_{1}^{2}$. The optimal $c_{0}=8$ is close to $c_{0}=7$ we found suitable in the case of pipe flow. Overall, the two-parameter ILU factorization leads to a more efficient preconditioner in terms of memory usage (fill-in) and iteration counts, but with a more expensive set-up stage, compared to the standard $I L U(\tau)$. 
TABLE 5

The performance of $\operatorname{ILU}(\tau)$ and $\operatorname{ILU}\left(\tau, c_{0} \tau^{2}\right)$ depending on the choice of threshold parameters for the Ethier-Steinman flow; results are shown for $\nu=0.001, \alpha=1$, mesh 3.

\begin{tabular}{crrr|rrrrr}
\hline$\tau$ & fill $_{L U}$ & \#it & $T_{\mathrm{CPU}}$ & $\tau$ & \multicolumn{1}{c}{$c_{0}$} & fill $_{L U}$ & $\#$ it & $T_{\mathrm{CPU}}$ \\
\hline \multicolumn{3}{c}{$\operatorname{ILU}(\tau)$} & \multicolumn{7}{c}{$\mathrm{ILU}\left(\tau, c_{0} \tau^{2}\right)$} \\
0.0065 & 76.041 & $\mathrm{n} / \mathrm{c}$ & & & & & & \\
0.0060 & 76.816 & 107 & 656.8 & 0.02 & 12.5 & 27.239 & $\mathrm{n} / \mathrm{c}$ & \\
0.0055 & 78.068 & 55 & 632.5 & 0.02 & 10 & 23.764 & 553 & 570.7 \\
0.0050 & 79.769 & 34 & 655.2 & 0.02 & 9 & 22.609 & 145 & 450.8 \\
0.0045 & 82.126 & 26 & 676.0 & 0.02 & 8 & 21.537 & 86 & 438.2 \\
0.0040 & 85.046 & 18 & 724.1 & 0.02 & 7.5 & 21.084 & 73 & 439.7 \\
0.0030 & 93.718 & 12 & 868.7 & 0.02 & 7 & 20.616 & 58 & 440.1 \\
0.0020 & 108.269 & 8 & 1135.7 & 0.02 & 6 & 19.963 & 50 & 448.8 \\
0.0015 & 119.858 & 7 & 1383.4 & 0.02 & 5 & 18.967 & 45 & 470.1 \\
0.0010 & 137.594 & 5 & 1781.1 & 0.02 & 4 & 18.108 & 39 & 508.4 \\
\hline
\end{tabular}
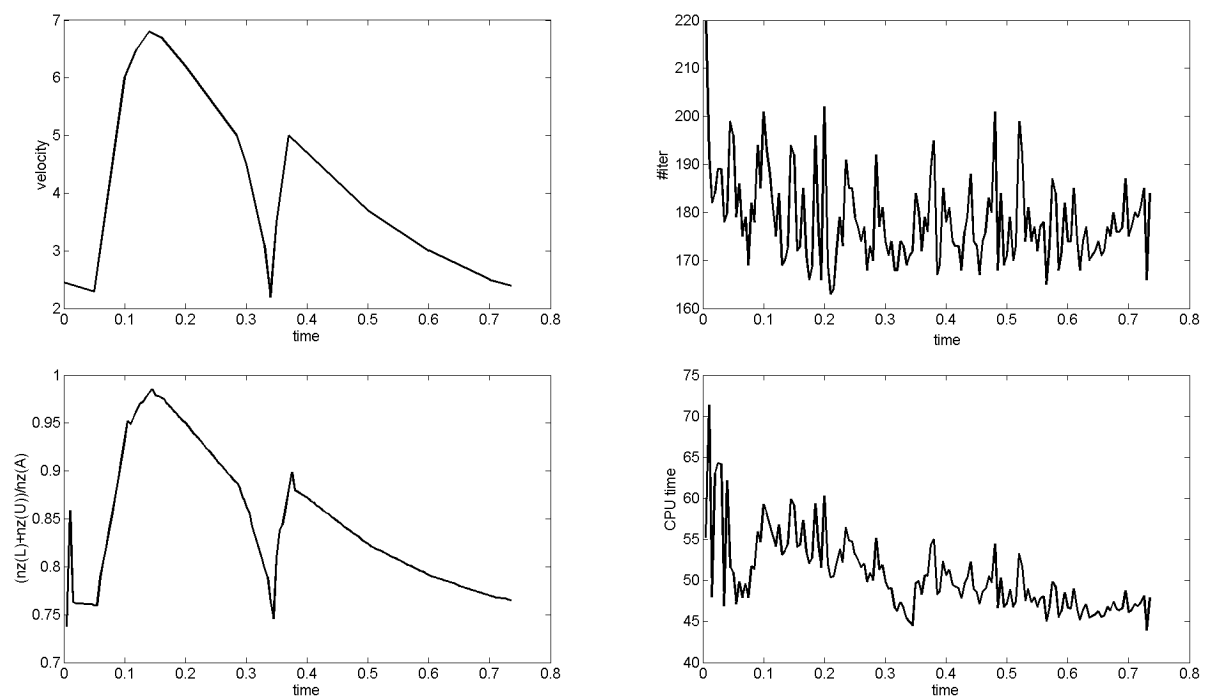

FIG. 4. Right coronary artery test case: The top-left plot shows the velocity waveform on the inflow, the top-right plot shows the number of BiCGStab iterations, the bottom-left plot shows the fill-in ratio, and the bottom-right plot shows linear system solution CPU times. All shown data are functions of time.

5.3. Flow in a right coronary artery. Finally, we study the performance of the ILU preconditioner for a model haemodynamic problem of blood flow in a right coronary artery. The geometry of the flow domain was recovered from a real patient coronary CT angiography, the ANI3D package [24] was used to generate the tetrahedral mesh (see Figure 5), and to build the FE systems (1.3). The diameter of the inlet cross section is about $0.27 \mathrm{~cm}$ and the whole domain can be embedded in a parallelogram with sides $6.5 \mathrm{~cm} \times 6.8 \mathrm{~cm} \times 5 \mathrm{~cm}$. The mesh consists of 120191 tetrahedra leading to the discrete Navier-Stokes system with 623883 unknowns. Other model parameters are $\nu=0.04 \mathrm{~cm}^{2} / \mathrm{s}, \rho=1 \mathrm{~g} / \mathrm{cm}$, one cardiac cycle period was $0.735 \mathrm{~s}$. The inlet velocity waveform is shown in Figure 4 (top left); it was suggested in [19] on the basis of clinical measurements. This waveform was used to define the flow rate through the inflow cross section, while for the inflow velocity profile we prescribed the Poiseuille flow. The Neumann boundary condition $-\nu(\nabla \mathbf{u}) \cdot \mathbf{n}+p \mathbf{n}=\mathbf{0}$ was imposed 
on all outflow boundaries. No elasticity model was used for the vessel walls, i.e., the walls were treated as rigid.

The Navier-Stokes system (1.1) was integrated in time using a semi-implicit second order method with $\Delta t=0.005$. The Oseen problem (1.2) was solved on every time step with the preconditioned BiCGstab method. The solution from the previous time step was used as the initial guess. For the preconditioner we used the two-parameter ILU factorization with the choice of parameters $\tau_{1}=0.03, \tau_{2}=7 \tau_{1}^{2}$. Recall that these are quasi-optimal parameters for pipe flows from section 5.1. This choice of the preconditioner and parameters results in stable computations over the whole cardiac cycle. The preconditioner performance data are shown in Figure 4. It is interesting to note that the graph of the fill-in rate for the LU-factors repeats the waveform of the inflow velocity remarkably well. Thanks to this adaptive feature of the threshold factorization, the variations of the iteration numbers and computational times per linear solve are rather modest; see the right plots in Figure 4. The computed solution was physiologically relevant; it is illustrated in Figure 5.

6. Closing remarks and conclusions. In this paper, we studied threshold ILU preconditioners for the discrete linearized Navier-Stokes system. Incomplete elementwise factorization preconditioners have a clear advantage of being rather insensitive to several factors, such as a choice of discretization, boundary conditions for governing PDEs, domain geometry, and flow directions, which otherwise influence the performance of many other algebraic solvers for the fluid dynamics problem. Furthermore, the presented method does not need a choice of subsolvers or inner iterations in contrast to many block preconditioners. It is well known that for discrete elliptic problems, ILU preconditioners do not scale optimally with respect to the number of unknowns. We observed this nonoptimality in our numerical experiments as well. However, in numerical experiments this mesh dependence was more pronounced for diffusion dominated flows and less evident when convection plays an important role. For 3D problems, when the number of grid refinement levels is not large, such a dependence can be an acceptable price for other robustness properties of the preconditioner.

Small values of viscosity parameters cause problems for most, if not all, known preconditioners for (1.3). Our results show that the threshold ILU is not an exception. At the same time, we found that the performance range with respect to $\nu$ of $\operatorname{ILU}(\tau)$ and, especially, of $\operatorname{ILU}\left(\tau_{1}, \tau_{2}\right)$ is rather impressive, and likely covers most laminar flows. Introducing subgrid models for higher Re numbers (e.g., turbulent) flows changes the discrete system and, since such models are commonly dissipative, this improves algebraic properties of the discrete system and should make the presented preconditioning also feasible. We observed such an improvement if SUPG stabilization is added to the FE formulation of the Ethier-Steinman problem for $\nu=10^{-3}$, but these extra results are not included in the report.

Incomplete threshold factorization is not a black-box method. A user should make at least a choice of threshold parameter(s), and many techniques have been suggested in the literature to improve the performance of ILU preconditioners. For fluid flows treated in this paper, we found that natural $\mathbf{u}-p$ ordering of unknowns and matrix two-sided scaling is sufficient for numerical stability. Further performance improvements by using matrix-band diminishing or streamwise (for transport dominated problems) reordering of velocity unknowns, can be possible. Although optimal threshold parameters appear to be flow dependent, quasi-optimal $\tau$ 's can be chosen and successfully used for a wide range of flow and discretization parameters. 

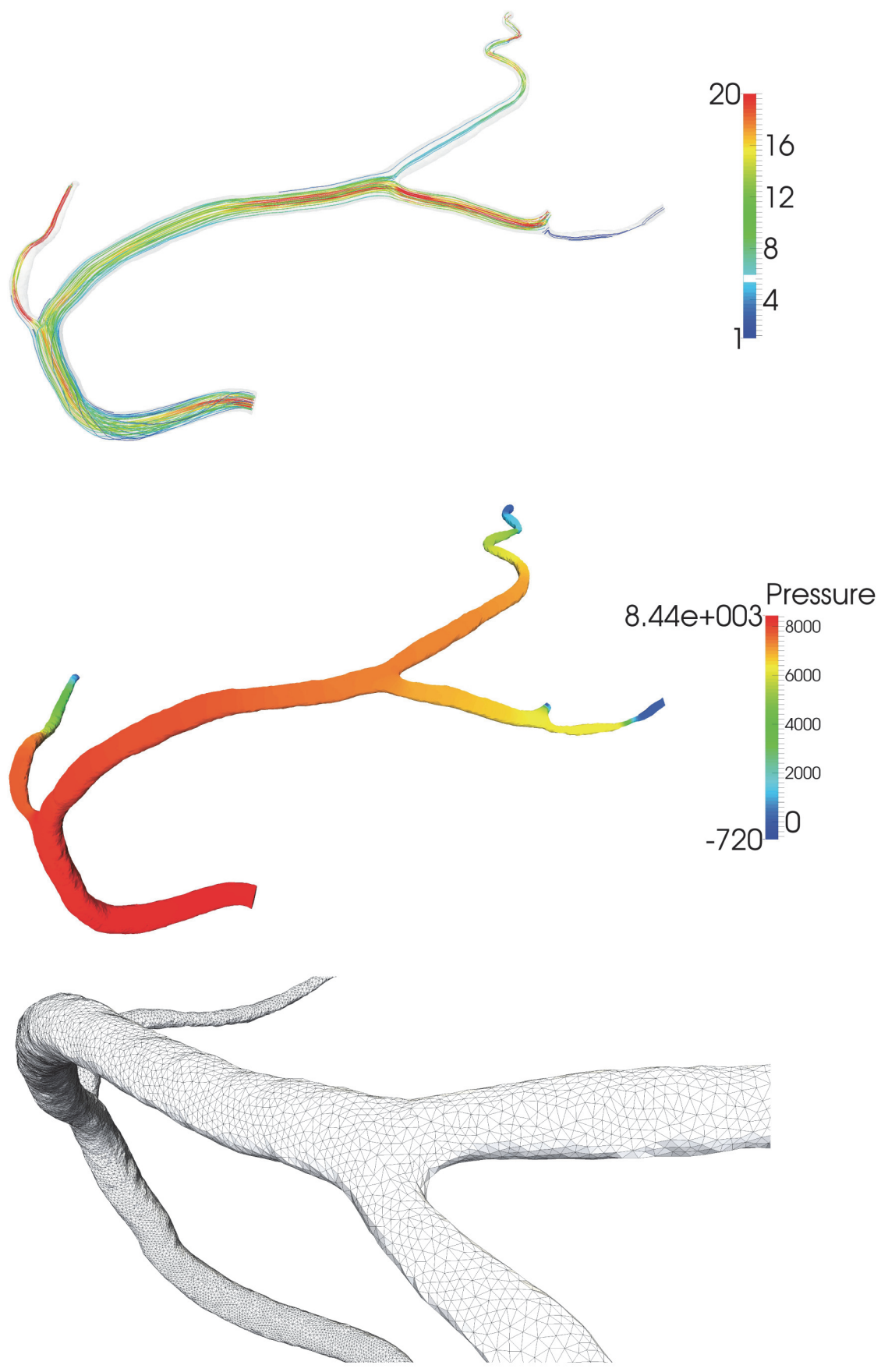

FIG. 5. Right coronary artery: Top picture shows selected steamlines colored by the velocity absolute value at time $0.4 \mathrm{~s}$; middle picture shows the transmural pressure distribution at time $0.4 \mathrm{~s}$; bottom picture illustrates the grid for this test case. 
We considered a Tismenetsky-Kaporin-type incomplete two-parameter factorization for nonsymmetric matrices and tested it for matrices arising in computational fluid dynamics. While for modest values of $\nu$ (leading to a parity between convection and diffusion terms) the performance of $\operatorname{ILU}\left(\tau_{1}, \tau_{2}\right)$ was similar to that of $\operatorname{ILU}(\tau)$, for larger and smaller $\nu$ 's $\operatorname{ILU}\left(\tau_{1}, \tau_{2}\right)$ was found to provide a more efficient preconditioner. It was observed that the $\operatorname{ILU}\left(\tau_{1}, \tau_{2}\right)$ preconditioner has a low fill-in and leads to faster convergent iterations for the expense of a more time consuming set-up phase. These properties may make it an ideal choice for time-dependent computations, when one can reuse a preconditioner over several time steps.

A numerical analysis of incomplete factorizations for nonsymmetric matrices is still limited. This paper proves numerical stability bounds for the exact LU factorization of nonsymmetric saddle-point matrices. We estimated the dependence of the constants in these bounds on the flow problem parameters. This might give some insight into the performance of incomplete factorizations applied to flow problems.

The two-parameter ILU preconditioner was applied to simulate a blood flow in a right coronary artery reconstructed from a real patient coronary CT angiography. We found the performance of the preconditioner satisfactory.

Acknowledgments. The authors thank Tatiana Dobroserdova and Alexander Danilov for assistance in building tetrahedral meshes and FE systems, and Sergei Goreinov for sharing his implementation of the rowwise variant of the $\operatorname{ILU}\left(\tau_{1}, \tau_{2}\right)$ factorization. We are grateful to Igor Kaporin for his insightful comments on a draft of this report.

\section{REFERENCES}

[1] M. Benzi, Preconditioning techniques for large linear systems: A survey, J. Comput. Phys., 182 (2002), pp. 418-477.

[2] M. BENZI, A generalization of the Hermitian and skew-Hermitian splitting iteration, SIAM Journal on Matrix Analysis and Applications, 31 (2009), pp. 360-374.

[3] M. Benzi, G. H Golub, And J. Liesen, Numerical solution of saddle point problems, Acta Numer., 14 (2005), pp. 1-137.

[4] M. BenzI, M. NG, Q. NiU, AND Z. WANG, A relaxed dimensional factorization preconditioner for the incompressible Navier-Stokes equations, J. Comput. Phys., 230 (2011), pp. 61856202.

[5] M. Benzi, M. A. OlshanskiI, And Z. WANG, Modified augmented Lagrangian preconditioners for the incompressible Navier-Stokes equations, Internat. J. Numer. Methods Fluids, 66 (2011), pp. 486-508.

[6] M. Braack, P. B. Mucha, and W. M. Zajaczkowski, Directional do-nothing condition for the Navier-Stokes equations, J. Comput. Math., 32 (2014), pp. 507-521.

[7] Z.-H. CAO, A class of constraint preconditioners for nonsymmetric saddle point matrices, Numer. Math., 103 (2006), pp. 47-61.

[8] O. Dahl and S. $\varnothing$. Wille, An ILU preconditioner with coupled node fill-in for iterative solution of the mixed finite element formulation of the $2 D$ and $3 D$ Navier-Stokes equations, Internat. J. Numer. Methods Fluids, 15 (1992), pp. 525-544.

[9] V. F. de Almeida, A. M. Chapman, and J. J. Derby, On equilibration and sparse factorization of matrices arising in finite element solutions of partial differential equations, Numer. Methods Partial Differential Equations, 16 (2000), pp. 11-29.

[10] S. Deparis, G. Grandperrin, and A. Quarteroni, Parallel preconditioners for the unsteady Navier-Stokes equations and applications to hemodynamics simulations, Comput. \& Fluids, 92 (2014), pp. 253-273.

[11] H. Elman AND D. Silvester, Fast nonsymmetric iterations and preconditioning for NavierStokes equations, SIAM Journal on Scientific Computing, 17 (1996), pp. 33-46.

[12] H. C. Elman, D. Silvester, And A. Wathen, Finite Elements And Fast Iterative Solvers: With Applications in Incompressible Fluid Dynamics, 2nd ed., Oxford University Press, Oxford, 2014. 
[13] H. C. Elman and R. S. Tuminaro, Boundary conditions in approximate commutator preconditioners for the Navier-Stokes equations, Electron. Trans. Numer. Anal., 35 (2009), pp. 257-280.

[14] C. R. Ethier And D. A. Steinman, Exact fully 3D Navier-Stokes solutions for benchmarking, Internat. J. Numer. Methods Fluids, 19 (1994), pp. 369-375.

[15] V. Girault and P.-A. Raviart, Finite Element Approximation of the Navier-Stokes Equations, Lecture Notes in Math., Springer-Verlag, Berlin, 1979.

[16] G. H. Golub and C. F. van Loan, Matrix Computations, Johns Hopkins University Press, Baltimore, 1996.

[17] G. H. Golub and C. VAN LoAn, Unsymmetric positive definite linear systems, Linear Algebra Appl., 28 (1979), pp. 85-97.

[18] J. Guzmán and M. NeIlan, Conforming and divergence-free Stokes elements in three dimensions, IMA J. Numer. Anal., 34 (2014), pp. 1489-1508.

[19] J. Jung, A. Hassanein, AND R. W. LyCZKOWski, Hemodynamic computation using multiphase flow dynamics in a right coronary artery, Ann. Biomed. Eng., 34 (2006), pp. 393-407.

[20] I. E. KAPORIN, High quality preconditioning of a general symmetric positive definite matrix based on its $U^{T} U+U^{T} R+R^{T} U$-decomposition, Numer. Linear Algebra Appl., 5 (1998), pp. 483-509.

[21] I. E. KAPORIN, Scaling, reordering, and diagonal pivoting in ILU preconditionings, Russian J. Numer. Anal. Math. Modelling, 22 (2007), pp. 341-375.

[22] I. E. KAPORIN, Scaling, preconditioning, and superlinear convergence in GMRES-type iterations, in Matrix Methods: Theory, Algorithms, Applications, V. Olshevsky and E. Tyrtyshnikov, eds., World Scientific Publ., Hackensack, NJ, 2010, pp. 273-295.

[23] K. Lipnikov and Yu. Vassilevski, Advanced Numerical Instruments 2D, http://sourceforge. net/projects/ani2d.

[24] K. Lipnikov and Yu. Vassilevski, Advanced Numerical Instruments 3D, http://sourceforge. net/projects/ani3d.

[25] O. E. Livne And G. H. Golub, Scaling by binormalization, Numer. Algorithms, 35 (2004), pp. $97-120$.

[26] J. MAYER, Symmetric permutations for i-matrices to delay and avoid small pivots during factorization, SIAM Journal on Scientific Computing, 30 (2008), pp. 982-996.

[27] M. A. Olshanskit And A. Reusken, Grad-div stablilization for Stokes equations, Math. Comput., 73 (2004), pp. 1699-1718.

[28] M. A. Ol'shanski And V. M. Staroverov, On simulation of outflow boundary conditions in finite difference calculations for incompressible fluid, Internat. J. Numer. Methods Fluids, 33 (2000), pp. 499-534.

[29] M. A. Olshanskit and E. E. Tyrtyshnikov, Iterative Methods For Linear Systems: Theory and Applications, Appl. Math. SIAM, Philadelphia, 2014.

[30] M. A. Olshanski And Y. V. VAssilevski, Pressure Schur complement preconditioners for the discrete Oseen problem, SIAM Journal on Scientific Computing, 29 (2007), pp. 2686-2704.

[31] Y. SAAD, Iterative Methods for Sparse Linear Systems, 2nd ed., SIAM, Philadelphia, 2003.

[32] R. L. Sani and P. M. Gresho, Résumé and remarks on the open boundary condition minisymposium, Internat. J. Numer. Methods Fluids, 18 (1994), pp. 983-1008.

[33] J. SCOTT AND M. TÜma, On signed incomplete Cholesky factorization preconditioners for saddle-point systems, SIAM Journal on Scientific Computing, 36 (2014), pp. A2984-A3010.

[34] J. Scott And M. Tuma, Solving Symmetric Indefinite Systems Using Memory Efficient Incomplete Factorization Preconditioners, Technical report RAL-P-2015-002, STFC Rutherford Appleton Laboratory, Chilton, England, 2015.

[35] A. Segal, M. ur Rehman, and C. Vuik, Preconditioners for incompressible Navier-Stokes solvers, Numer. Math. Theory Methods Appl., 3 (2010), pp. 245-275.

[36] G. W. Soules, The rate of convergence of Sinkhorn balancing, Linear Algebra Appl., 150 (1991), pp. 3-40.

[37] J. Stoer And R. Bulirsch, Introduction to Numerical Analysis, Springer, New York, 1993.

[38] M. Suarjana And K. H. LaW, A robust incomplete factorization based on value and space constraints, Internat. J. Numer. Methods Engrg., 38 (1995), pp. 1703-1719.

[39] R. Temam, Navier-Stokes Equations: Theory and Numerical Analysis, American Mathematical Soc., Providence, RI, 2001.

[40] M. Tismenetsky, A new preconditioning technique for solving large sparse linear systems, Linear Algebra Appl., 154 (1991), pp. 331-353.

[41] S. Turek, Efficient Solvers for Incompressible Flow Problems: An Algorithmic and Computational Approache, 6, Springer Lecture Notes Comput. Sci. Eng., Berlin, 1999. 
[42] M. ur Rehman, C. Vuik, AND G. Segal, A comparison of preconditioners for incompressible Navier-Stokes solvers, Internat. J. Numer. Methods Fluids, 57 (2008), pp. 1731-1751.

[43] M. ur Rehman, C. Vuik, and G. Segal, Simple-type preconditioners for the Oseen problem, Internat. J. Numer. Methods Fluids, 61 (2009), pp. 432-452.

[44] M. WABro, AMGe-coarsening strategies and application to the Oseen equations, SIAM Journal on Scientific Computing, 27 (2006), pp. 2077-2097.

[45] J. ZHAO, The generalized Cholesky factorization method for saddle point problems, Appl. Math. Comput., 92 (1998), pp. 49-58. 